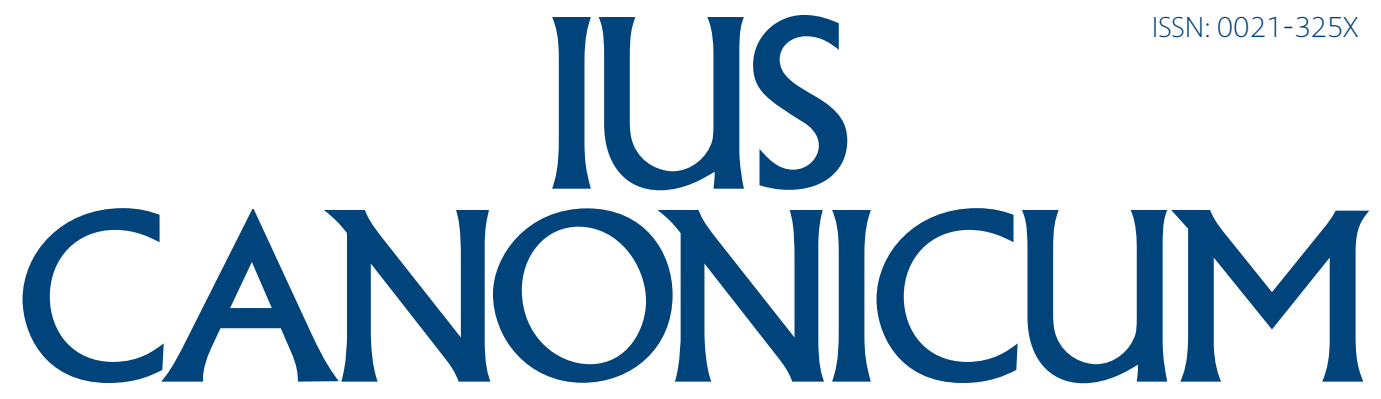

REVISTA DEL INSTITUTO MARTÍN DE AZPILCUETA

FACULTAD DE DERECHO CANÓNICO

UNIVERSIDAD DE NAVARRA

VOLUMEN 58
NÚMERO 116

DICIEMBRE 2018 


\section{IUS CANONICUM}

REVISTA SEMESTRAL DEL INSTITUTO MARTÍN DE AZPILCUETA / FACULTAD DE DERECHO CANÓNICO DE LA UNIVERSIDAD DE NAVARRA / PAMPLONA. ESPAÑA / FUNDADA EN 1961 / ISSN: 0021-325X DICIEMBRE 2018 / VOLUMEN 58

DIRECTOR / EDITOR

Jorge Otaduy UNIVERSIDAD DE NAVARRA jorotaduy@unav.es

CONSEJO EDITORIAL EDITORIAL BOARD

VOCALES

Daniel Cenalmor UNIVERSIDAD DE NAVARRA

Alejandro González-Varas UNIVERSIDAD DE ZARAGOZA

Julián Ros Córcoles

VICARIO JUDICIAL. ALBACETE

Joaquín Sedano

UNIVERSIDAD DE NAVARRA

Ana $M^{\mathrm{a}}$ Vega Gutiérrez UNIVERSIDAD DE LA RIOJA

\section{SECRETARIO}

Eduardo Flandes

UNIVERSIDAD DE NAVARRA

eflandes@unav.es

CONSEJO ASESOR / ADVISORY BOARD

Nicolás Álvarez

de las Asturias

UNIVERSIDAD ECLESIÁSTICA

SAN DÁMASO,

MADRID (ESPAÑA)

Juan Ignacio Arrieta

PONTIFICIO CONSIGLIO PER I TESTI

LEGISLATIVI, ROMA (CIUDAD DEL

VATICANO)

Orazio Condorelli

UNIVERSITÀ DEGLI STUDI DI

CATANIA (ITALIA)

Myriam Cortés

UNIVERSIDAD PONTIFICIA

DE SALAMANCA (ESPAÑA)

Brian Ferme

CONSIGLIO PER L'ECONOMIA, ROMA (CIUDAD DEL VATICANO)

Ombretta Fumagalli Carulli

UNIVERSITÀ CATTOLICA DEL

SACRO CUORE, MILANO (ITALIA)

Alberto de la Hera

UNIVERSIDAD COMPLUTENSE

DE MADRID (ESPAÑA)

\section{Luis Navarro \\ UNIVERSITÀ PONTIFICIA DELLA \\ SANTA CROCE, ROMA (ITALIA) \\ Rafael Navarro-Valls \\ UNIVERSIDAD COMPLUTENSE \\ DE MADRID (ESPAÑA)}

\section{María Elena Olmos \\ UNIVERSIDAD DE VALENCIA (ESPAÑA)}

\section{Carmen Peña \\ UNIVERSIDAD PONTIFICIA COMILLAS, MADRID (ESPAÑA)}

\section{Helmut Pree}

LUDWIG-MAXIMILIANS-UNIVERSITÄT, MÜNCHEN (ALEMANIA)

\section{María José Roca \\ UNIVERSIDAD COMPLUTENSE \\ DE MADRID (ESPAÑA)}

Antoni Stankiewicz

TRIBUNALE DELLA ROTA ROMANA, ROMA (ITALIA)

José María Vázquez

García-Peñuela

UNIVERSIDAD INTERNACIONAL

DE LA RIOJA (ESPAÑA)

Ius Canonicum es una revista general de Derecho canónico y de Derecho eclesiástico. Publica artículos científicos sometidos a revisión por expertos relativos a todos los sectores del ordenamiento canónico así como también los textos legislativos promulgados por la Santa Sede, la actividad del CPTL, y sentencias de los Tribunales de la Santa Sede, oportunamente comentados. Completan cada volumen la sección de recensiones y de crónicas de jurisprudencia, de legislación y de reuniones científicas.

Ius Canonicum is a journal of canon law and ecclesiastical law which publishes peer-reviewed articles written by experts on all matters relating to canon law and the legislative texts issued by the Holy See, the actions of the CPTL, and the sentences handed down by the tribunals of the Holy See, annotated as appropriate. The final section of each edition comprises a set of reviews and reports on jurisprudence, legislation and academic congresses. 


Redacción y
Administración
Instituto «Martín
de Azpilcueta»
Universidad de Navarra
31009 Pamplona
(España)
T948 425600
F948 425633
spublicaciones@unav.es
www.unav.es/ima

\section{Suscripciones}

spublicaciones@unav.es

\section{Edita}

Servicio de Publicaciones de la Universidad de Navarra

\section{Precios}

Edición impresa:

- Suscripciones para España y Unión Europea: 2 números al año, 57 € (IVA incluido)

- Suscripciones para otros países: 2 números al año, $70 €$

Edición electrónica: $40 €$

Fotocomposición

NovaText

Imprime

GraphyCems

D.L. NA 197-1961

Periodicidad

Semestral

Junio y diciembre

Tamaño $170 \times 240$ mm
Ius Canonicum figura en los siguientes índices y bases de datos:

- SCOPUS.

- HEINONLINE.

- ERIH Plus (European Reference Index for the Humanistics and Social Sciences).

- CNKI (China National Knowledge Infrastructure).

- ACADEMIC SEARCH COMPLETE (EBSCO).

- FRANCIS (Institute d'Information Scientifique, Centre National de la Recherche Scientifique, FR).

- PERIODICAL INDEX ONLINE (Pro-Quest, GB).

- ISOC, CIENCIAS SOCIALES Y HUMANIDADES (Centro de Ciencias Humanas y Sociales del CSIC, ES).

- CANON LAW ABSTRACTS (Canon Law Society of Great Britain and Ireland).

- RELIGIOUS AND THEOLOGICAL ABSTRACTS (William Sailer, USA).

- DIALNET (Universidad de La Rioja, ES).

- ATLA Religion Database.

Ius Canonicum figura en los siguientes rankings y clasificaciones de revistas:

- RESH (Sistema de valoración integrada de revistas españolas de Humanidades y Ciencias Sociales).

- CIRC (Clasificación integrada de revistas científicas).

- CARHUS Plus Agència de Gestió d'Adjuts Universitaris y de Rècerca.

- IN-RECJ (Índice de impacto de las revistas españolas de ciencias jurídicas).

- DICE (Difusión y Calidad Editorial de las Revistas Españolas de Humanidades y Ciencias Sociales y Jurídicas).

- Directorio y Catálogo LATINDEX (Sistema regional de información en línea para revistas científicas de América Latina, el Caribe, España y Portugal).

El contenido de lus Canonicum es accesible en formato electrónico en el sitio web de la revista (https://www.unav.edu/ius-canonicum) y en Dadun, repositorio de la Universidad de Navarra (http://dadun.unav.edu/handle/10171/3218), con un periodo de embargo para los números más recientes.

Las opiniones expuestas en los trabajos publicados por la revista son de la exclusiva responsabilidad de sus autores. 


\section{IUS CANONICUM}

DICIEMBRE 2018 / VOLUMEN 58

A PROPÓSITO DEL SIINODO SOBRE LOS JÓVENES 2018

Nicolás ÁlVAREZ DE LAS ASTURIAS

Discernir la idoneidad para el presbiterado: La contribución del derecho y de la tradición canónica latina al Sínodo de Obispos sobre los jóvenes

Discerning Suitability for Priesthood: The Contribution of Canon Law and the Latin $461-478$

Canonical Tradition to the Synod of Bishops on Young People

\section{ESTUDIOS / ARTICLES}

Thierry SoL

¿Una alternativa a la noción de executio potestatis? La separación entre potestas ordinis y officium según Hugo de Amiens y

Gerhoch de Reichersberg

An Alternative to the Notion of executio potestatis? The Separation Between

potestas ordinis and officium in Hugh of Amiens and Gerhoch of Reichersberg

Massimo DEL Pozzo

Chiarimenti pontifici sul "processus brevior". Riflessioni alla luce del Discorso del 25 novembre 2017

Pontifical Clarifications Regarding the "processus brevior". Reflections in Light

of the Address on November 25, 2017

Jorge CASTRo TRAPOTE

La mayoría de edad como presunción iuris tantum de capacidad en los códigos civiles y canónicos

The Age of Majority as a Presumption iuris tantum of Capacity in Civil and Canonical Codes 
Geraldina BONI - Manuel GANARIN

In merito al problema se i Superiori maggiori degli istituti

religiosi di diritto pontificio clericali possano erigere pie

fondazioni autonome

Regarding whether the Major Superiors of Clerical Religious Institutes of Pontifical

$581-610$

Right Can Establish Autonomous Pious Foundations

Luis NAVARRO

Nuevos movimientos eclesiales. Naturaleza de los carismas, cuestiones jurídicas y límites

New Ecclesial Movements. The Nature of Charisms, Legal Concerns and Limits

Javier OTADUY GUERÍN

Unidad y pluralidad en la Iglesia. El pluralismo carismático

Unity and Plurality in the Church. Charismatic Pluralism

Jorge Salinas Mengual

Protección de datos: entre el derecho a la intimidad y

la autonomía de las confesiones religiosas. El caso finlandés y el español (a propósito de la Sentencia Jehovan Todistajat del TJUE)

Data Protection: Between the Right to Privacy and the Autonomy of Religious

Confessions. The Finnish and Spanish Case (Concerning the CJEU Judgement on Jehovan Todistajat)

Antonio VIANA

Introducción histórica y canónica al oficio eclesiástico

Historical and Canonical Introduction to the Ecclesiastical Office

$671-708$

Francisco José Zamora García

Iglesia y Estado en el constitucionalismo isabelino

Church-State Relations in the Reign of Isabel II

Ciro TAMMARO

L'instructio probatoria nel processo penale medievale:

osservazioni canoniche sull'ammissione e l'assunzione

dei mezzi di prova nei secoli XIII e XIV

Instructio probatoria in Medieval Penal Trial: Canonical Observations about

the Admission and the Experiment of the Evidences in XIII and XIV Centuries

COMENTARIOS Y NOTAS / COMMENTS AND NOTES

Bruno Esposito, O. P.

Presentación y comentario de la Constitución Apostólica

Veritatis gaudium y de las Ordinationes anejas,

sobre las Universidades y Facultades eclesiásticas

$813-856$ 


\section{BIBLIOGRAFÍA / BOOK REVIEWS}

\section{RECENSIONES}

Asensio SÁnchez, M. Á. - Calvo Espiga, A. - Menéndez-ValdéS

Navas, M. - Parody Navarro, J. A., Fenómeno religioso y ordenamiento

jurídico (J. Ferrer ORTIZ)

CELADOR ANGÓN, Ó., Orígenes histórico constitucionales del principio

de laicidad (J. Ma VázQuez GarCíA-PeÑuelA)

Daniel, W. L., The Art of Good Governance. A Guide to the Administrative

Procedure for Just Decision-Making in the Catholic Church (J. MIRAS)

FERnánDEZ SAN ROMán, J., La relevancia del abandono de la fe y de la condición de censurado en la admisión al matrimonio. Estudio del "iter" redaccional de los cánones 1065 y 1066 en la Codificación de 1917 y de las demás fuentes hasta el Concilio Vaticano I/ (C. GARCIMARTín)

García y García, A. (dir.); CANTElar RodrígueZ, F. - García y

García, A. (†) - García Matamoro, L. A. - Justo Fernández, J. -

MArquès Sala, B. (eds.), Synodicon Hispanum. XIII. Ager (Abadía), Barcelona,

Lérida, Segorbe-Albarracín y Urgell (J. SEDANO)

$874-876$

MANTECÓN SANCHO, J., Religión, valores y libertad religiosa. Una visión personal (J. FerRer OrTiz)

Martí SÁnchez, J. Ma - Moreno Mozos, Ma del M. (coords.), Derecho de difusión de mensajes y libertad religiosa (R. PALOMINo LOZANO)

Melville, G. - Helmrath, J. (eds.), The Fourth Lateran Council.

Institutional Reform and Spiritual Renewal (J. SEDANO)

NúÑEZ RIVERO, C., La religión católica en la historia política y constitucional española (1808-1931) (Ma del M. Martín GarCía)

Pelayo Olmedo, J. D., Una nueva regulación del Registro de Entidades

Religiosas. Entre el control y la gestión de la libertad en el tratamiento

de la diversidad religiosa (R. PALOMINO LOZANO)

PÉrez-MAdrid, F. (coordinadora), Religión, libertad y seguridad (J. Ferrer OrTiz) 896-900 RocA, $M^{\mathrm{a}} \mathrm{J}$., La libertad religiosa negativa. La apostasía en el Derecho confesional y comparado (J. OTADUY)

Ruano Espina, L. - SÁnchez Girón, J. L. (eds.), Novedades de Derecho

Canónico y Derecho Eclesiástico del Estado. A un año de la reforma del proceso matrimonial (J. A. FuENTES ALONSO)

TAMmLER, U., Albert Michael Koeniger (1874-1950). Aus dem Leben und Wirken eines schwäbisch-bayerischen Kanonisten und Kirchen(rechts)historikers (A. DE LA HERA) 907-911 


\title{
In merito al problema se i Superiori maggiori degli istituti religiosi di diritto pontificio clericali possano erigere pie fondazioni autonome*
}

\author{
Regarding whether the Major Superiors of Clerical \\ Religious Institutes of Pontifical Right Can Establish \\ Autonomous Pious Foundations
}

RECIBIDO: 16 DE JUNIO DE 2018 / ACEPTADO: 1 DE AGOSTO DE 2018

\section{Geraldina BONI}

Professoressa ordinaria Dipartimento di Scienze Giuridiche

Università di Bologna. Bologna

orcid 0000-0003-2098-8469

geraldina.boni@unibo.it

\section{Manuel GANARIN}

Professore a contratto Dipartimento di Scienze Giuridiche Università di Bologna. Bologna orcid 0000-0002-0323-852X manuel.ganarin2@unibo.it

Resumen: El artículo examina los diferentes aspectos de una quaestio iuris que es particularmente actual y discutida entre los canonistas, con respecto a la posibilidad de que los Superiores mayores de los institutos religiosos clericales de derecho pontificio erijan fundaciones pías autónomas con personalidad jurídica canónica pública o privada. A la luz de la legislación universal y particular en vigor, los criterios generales para la interpretación del derecho canónico y la práctica, el trabajo intenta ofrecer una solución a esta cuestión.

Palabras clave: Fundaciones pías autónomas, Superiores mayores, Institutos religiosos clericales de derecho pontificio.
Abstract: The paper examines various aspects of a quaestio iuris of current pressing concern among canonists, regarding whether the major superiors of clerical religious institutes of pontifical right may set up autonomous pious foundations of a public or private canonical, legal nature. The purpose of this paper is to offer a solution to this matter, on the basis of the universal and particular laws in force, the general criteria that shape their interpretation and the practice of canon law.

Keywords: Autonomous Pious Foundations, Major Superiors, Clerical Religious Institutes of Pontifical Right.

* Geraldina Boni è autrice dei paragrafi 1, 2, 3.6, 3.7, 3.8 e 3.9. Manuel Ganarin è autore dei paragrafi 3.1, 3.2, 3.3, 3.4, 3.5 e 3.10. Il paragrafo 4 è comune. 


\section{LA QUAESTIO IURIS}

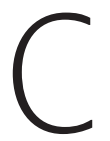

ome noto, in base al can. $1303, \$ 1,1$, gli elementi che integrano una fondazione autonoma sono: un insieme di beni o patrimonio fondazionale, cioè la massa bonorum -res materiali ma anche spirituali ${ }^{1}$ : cfr. can. $115, \$ 3$ - che deve produrre i frutti da impiegare per l'espletamento dell'attività e che viene eretta in persona giuridica canonica; la finalità ecclesiale (cfr. can. 114, \$2); l'erezione da parte dell'autorità ecclesiastica in forza della quale la fondazione è costituita e acquista personalità giuridica con decreto, diventando nuovo centro di imputazione di rapporti giuridici. Come ogni persona giuridica, anche le fondazioni autonome devono avere propri statuti approvati dalla competente autorità ecclesiastica (can. 117) che ha eretto l'ente.

Un tema dibattuto di particolare interesse sul quale si è di recente soffermata la canonistica -con numerose e incisive proiezioni pratiche- concerne la possibilità per i Superiori maggiori degli istituti di diritto pontificio clericali -ordinari, a norma del can. 134, $\$ 1$, nonché titolari della potestas regiminis in forza del can. 596, $\$ 2$ - di erigere fondazioni autonome con personalità giuridica pubblica o privata. Il presente studio intende esaminare $\mathrm{i}$ profili sottesi a tale quaestio iuris esplorati dalla canonistica, nel tentativo di offrire una soluzione alla luce sia della normativa vigente sia dei criteri generali di interpretazione del diritto canonico.

\section{LE ARGOMENTAZIONI A SOSTEGNO DELLA TESI NEGATIVA ADDOTTE DA EMINENTE DOTTRINA}

Già in passato un illustre canonista e cardinale, da poco scomparso, Velasio de Paolis ${ }^{2}$, ha concentrato ampiamente la sua attenzione sul quesito sopra enunciato, giungendo alla conclusione che i Superiori maggiori ${ }^{3}$ degli istituti di diritto pontificio clericali non sono titolari del potere esecutivo per erigere pie fondazioni autonome.

\footnotetext{
${ }^{1}$ Si veda recentemente J. MiÑAMBrEs, Fondazioni pie e figure affini, Ius Ecclesiae 21 (2009) 333 ss.

2 Cfr. V. DE PAOLIS, La vita consacrata nella Chiesa, edizione rivista e ampliata a cura di V. MosCA, Marcianum Press, Venezia 2010, 128 ss. (si tratta della riproposizione e rielaborazione di articoli precedenti: L'autorità competente ad erigere una persona giuridica nella Chiesa, Informationes SCRIS 26 [2000] 59 ss.; Periodica 92 [2003] 3 ss.; 223 ss.).

${ }^{3}$ Per l'identificazione dei Superiori maggiori cfr. can. 620.
} 
Sintetizzando le ragioni giuridiche prospettate esaustivamente dall'Autore, si deve constatare anzitutto come sia in qualche modo dirimente la circostanza che nel vigente Codex Iuris Canonici manchino norme che autorizzino l'erezione di fondazioni autonome (pubbliche o private) da parte degli ordinari, tra i quali sono da computarsi, per il can. 134, $\$ 1$, i Superiori maggiori degli istituti religiosi di diritto pontificio clericali e delle società di vita apostolica di diritto pontificio clericali.

È vero che nel Codice del 1983 il can. 1489 della codificazione pianobenedettina, il quale parlava in modo espresso della competenza unicamente dell'ordinarius loci per l'erezione di «instituta» (istituti ecclesiastici non collegiali ${ }^{4}$ ), non è più presente. V'era nell'abrogato Codice la possibilità che istituti religiosi avessero il privilegio apostolico di erigere tali «instituta» (cann. 1491, $\$ 1$ e 1492, $\$ 1$ ): quindi esclusivamente con l'intervento della Santa Sede. Ma sul punto -nonostante le consistenti innovazioni introdotte circa il regime delle persone giuridiche- la disciplina non è mutata. L'estensione di tale competenza agli ordinari non può infatti in alcun modo desumersi dal silenzio: occorre una previsione esplicita ed inequivoca del diritto universale della Chiesa. Vi fu la proposta durante i lavori preparatori postconciliari di ripristinare i cann. 1489 e 1491 ed essa fu respinta: non peraltro con la motivazione di sovvertire la disciplina, ma eccependo che questa si trovava altrove nel reformando Codice («Consultores respondent provisiones circa materiam illorum canonum haberi in aliis partibus novi codicis» $\left.{ }^{5}\right)$. La regolamentazione dell'erezione di associazioni nel Codex Iuris Canonici del 1917 è quindi, a parere di De Paolis, una riprova indiretta dell'esclusione della competenza dei semplici ordinari, quali i Superiori religiosi, competenza invero rigorosamente attribuita al solo Sommo Pontefice e agli ordinari del luogo (cfr. can. 686, $\$ 2$ della codificazione pianobenedettina).

Quanto alla potestà di erigere fondazioni il Codice del 1983 tace, come si è visto, sul punto, né fa alcuna distinzione tra persone giuridiche pubbliche e private. La creazione di entrambe, tuttavia, non avrebbe senso per gli

${ }^{4}$ Cfr. M. López Alarcón, sub can. 1303, en J. I. Arrieta (ed.), Codice di diritto canonico e leggi complementari commentato, $5^{\text {a }}$ ed., Coletti a San Pietro, Roma 2015, 868.

5 Pontificia Commissio Codici Iuris Canonici Recognoscendo. Coetus studiorum «De BONIS ECCLESIAE TEMPORALIBUS», Sessio II (diebus 12-16 novembris 1979 babita). Adunatio diei 15 novembris 1979, Communicationes 12 (1980) 435. 
istituti religiosi, esorbitando dal loro ambito. D'altronde «se si dovessero creare fondazioni di valore puramente interno all'istituto, non si vede quale utilità esse potrebbero avere, in quanto fa già da sufficiente supporto la personalità giuridica dell'istituto o della provincia o della casa» ${ }^{6}$; poi «che senso possono avere all'interno dell'ordinamento canonico fondazioni autonome private da parte di un istituto religioso, i cui beni sono ecclesiastici, perché persone giuridiche pubbliche?» ${ }^{7}$. In quest'ultimo caso, inoltre, la perdita della qualità di beni ecclesiastici, e dunque la sottrazione alla regolazione canonica di cui al Libro $\mathrm{V}$ del Codex, potrebbe essere inopportuna se non assai rischiosa.

Il potere poi di erigere universitates rerum (ed anche ovviamente universitates personarum) quali persone giuridiche afferisce al potere giurisdizionale in foro esterno e al governo della compagine ecclesiale: esso, nonostante il can. $596, \$ 2^{8}$, non spetta ai Superiori degli istituti religiosi, i quali non appartengono alla struttura gerarchica della Chiesa. D'altra parte si prevede che «quando il Superiore religioso vuole costituire delle comunità in una diocesi ha bisogno del consenso del Vescovo diocesano: esse non vengono erette in persona giuridica dal Superiore, ma dallo stesso ordinamento giuridico (can. $609 \$ 1){ }^{9}$. Tale disposizione e la speculare assenza di un disposto ove si contempli un qualche intervento dell'autorità gerarchica in caso di erezione di persone giuridiche da parte del Superiore religioso conferma la non sussistenza di tale facoltà.

Erigere fondazioni pie autonome che operano nella Chiesa e nella diocesi, esplicando comunque forme di apostolato nella vita ecclesiale, eccede il potere di governo dei religiosi che si esercita solo sull'istituto e non può interferire con le potestà della gerarchia ecclesiastica. I poteri che ha l'ordinario religioso sono quelli che gli conferisce tassativamente la legge canonica in funzione della vita e del carisma dell'istituto. E il diritto codiciale non gli accorda quello de quo.

Il Concilio Vaticano II ha indubbiamente valorizzato la dimensione ecclesiale degli istituti religiosi ed esaltato la loro giusta autonomia di vita e

\footnotetext{
${ }^{6}$ V. DE PAOLIS, La vita consacrata nella Chiesa, cit., 129.

V. DE PAOLIS, La vita consacrata nella Chiesa, cit., 129.

${ }^{8}$ Sulla novità di questa previsione rispetto alla disciplina precedente cfr. T. RINCÓN-PÉREZ, sub can. 596, en Á. Marzoa - J. Miras - R. Rodríguez-OCaÑa (eds.), Comentario exegético al Código de Derecho Canónico, II/2, $3^{\text {a }}$ ed., Eunsa, Pamplona 2002, 1473 ss.

${ }^{9}$ V. DE PAOLIS, La vita consacrata nella Chiesa, cit., 131.
} 
di governo: ma non li ha in alcun modo assimilati alle Chiese particolari. Essi, sottolinea De Paolis, non fanno in quanto tali parte della struttura gerarchica ecclesiale (cfr. Costituzione dogmatica Lumen gentium, n. 44) ${ }^{10}:$ «La categoria degli istituti di diritto pontificio clericali, se hanno questo riconoscimento, è in funzione della loro peculiare indole clericale, in funzione del carisma, non in funzione di una realtà gerarchica come sono gli ordinari locali o diocesani. Se i Superiori maggiori degli istituti di diritto pontificio clericali hanno un potere anche di governo e sono anche ordinari (can. 134) ciò non significa che abbiano nella Chiesa tutte le competenze per le quali basti il potere di governo anche solo esecutivo. Il principio generale è che i Superiori religiosi hanno solo quelle competenze che il diritto conferisce loro espressamente» ${ }^{11}$.

${ }^{10}$ Si veda tuttavia l'approfondita analisi, corredata di un'illustrazione sintetica dell'evoluzione storica, di B. EsposiTo, Alcune riflessioni sul Superiore maggiore in quanto Ordinario e sulla valenza ecclesiologica e canonica della qualifica, Angelicum 78 (2001), in particolare 727-729, il quale procedendo dalla lettura del can. 586, che 'riconosce' (agnoscitur) -e perciò non 'conferisce'- l'autonomia di vita, e soprattutto di governo, degli istituti religiosi, e avendo presente quanto stabilito dai cann. $134, \S 1$ e $596, \S 2$, conclude che la figura del Superiore maggiore degli istituti di vita consacrata e delle società di vita apostolica clericali di diritto pontificio «realizza perfettamente vista la natura di questi istituti, la figura di ufficio ecclesiastico di governo, ed in quanto tale appartiene all'organizzazione gerarchica della Chiesa. Attraverso loro si realizza a pieno titolo una partecipazione della vita consacrata alla struttura gerarchica della Chiesa (cfr. can. 207, $\$ \$ 1-2)$. Stabilire chiaramente qual è il posto occupato dal Superiore maggiore in quanto Ordinario e quali siano le sue relazioni con le altre autorità facenti parte della organizzazione ecclesiastica è terreno per ulteriori approfondimenti» (ivi, 729: peraltro Esposito, in precedenza, non menziona l'erezione di persone giuridiche tra i doveri specifici dei Superiori maggiori 'Ordinari' né tra i loro diritti specifici). E comunque tale Autore conclude: «In ogni caso, avere determinato con certezza che l'ufficio del Superiore maggiore in quanto Ordinario è un vero e proprio ufficio ecclesiastico, partecipe dell'unica potestà che Cristo ha dato alla Sua Chiesa, ricorda a tutti che questa autorità, ed i poteri di cui è dotata, hanno senso se vissuti ed esercitati nella Chiesa e per la Chiesa. Quindi non in modo assoluto, indipendente e competitivo, ma in spirito di servizio della Chiesa, nella continua ricerca della volontà di Dio» (ivi, 731). Cfr. per converso D. ANDRÉs, Le forme di vita consacrata. Commentario teologico-giuridico al Codice di Diritto Canonico, $6^{a}$ ed., Ediurcla, Roma 2008, 78-79, il quale, nel commentare il can. 596, $\$ 2$, secondo cui i Superiori degli istituti religiosi clericali di diritto pontificio godono inoltre della potestà ecclesiastica di governo, tanto per il foro esterno quanto per quello interno, evidenzia che tale potestà «è quella di governo o regime ecclesiastico di cui parla il can. 129, cioè, propria ed esclusiva della gerarchia della Chiesa, per la cui abilitazione e possesso è necessario essere ordinato in sacris». Peraltro lo stesso Autore, Los Superiores religiosos de los religiosos según el Código: IV Estatuto específico de los Superiores mayores Ordinarios, Commentarium pro religiosis et missionariis 79 (1998) 161 ss., distingue tra ordinari in senso proprio e pieno e ordinari in senso proprio ma limitato, tra cui appunto i Superiori maggiori di istituti religiosi clericali di diritto pontificio o di società di vita apostolica clericali di diritto pontificio.

${ }^{11}$ V. DE PAOLIS, La vita consacrata nella Chiesa, cit., 140-141. 
Muovendo pertanto dalle considerazioni appena concisamente descritte, Velasio de Paolis sostiene in conclusione che i Superiori maggiori degli istituti di diritto pontificio clericali non siano titolari della potestà esecutiva per erigere pie fondazioni autonome: «Perché i Superiori possano creare altri soggetti giuridici dei beni ecclesiastici, che per ipotesi dovrebbero essere pubblici, dovrebbero avere una facoltà, concessa come privilegio o in altro modo, come attraverso una norma costituzionale approvata dall'autorità competente» ${ }^{12}$. Dunque, oltre alla concessione per privilegio, la creazione di nuovi soggetti giuridici da parte dei Superiori maggiori potrebbe essere regolata nelle Costituzioni sempre approvate dalla Sede Apostolica ${ }^{13}$. Per converso, il Codice conferisce ai Superiori religiosi in quanto ordinari (can. 134, $\$ 1$ ) soltanto la possibilità di accettare fondazioni non autonome, cioè beni temporali comunque devoluti ad una persona giuridica pubblica (cfr. cann. 1303, $\$ 1,2$ e 1304, $\$ 1$ ).

$\mathrm{Al}$ di là della sfera prettamente giuridica, sembra che pure le ragioni per così dire più sostanziali e pratiche alla base della riserva dell'erezione di universitates rerum quali persone giuridiche a favore della Santa Sede (ovvero del Vescovo diocesano o delle Conferenze Episcopali ${ }^{14}$ ) abbiano una certa consistenza alla luce di una prassi insediatasi negli ultimi decenni, peculiarmente per fare fronte alle difficoltà incontrate da non pochi istituti religiosi nella società odierna ${ }^{15}$. In particolare talora si è assistito alla tendenza da parte dei Superiori religiosi, oltre che a ricorrere a modelli civilistici, a mirare ad erigere direttamente fondazioni pie autonome con l'intento e la speranza di risolvere i problemi che devono attualmente affrontare gli istituti, nella crisi di vocazioni e dunque nella scarsità di personale, e dinanzi ad alcune linee di sviluppo congiunturali e dell'economia contemporanea. I rischi che si profilano investono però la salvaguardia dell'identità cristiana cattolica e la tutela del carisma proprio e della spiritualità degli istituti religiosi, lo scongiurare la dissipazione di beni ecclesiastici e comunque la preservazione dei fini a cui essi devono essere destinati: beni ecclesiastici -non va mai trascurato- strettamente legati alla na-

${ }^{12}$ V. DE PAOLIS, La vita consacrata nella Chiesa, cit., 130.

13 V. DE PAolis, La vita consacrata nella Chiesa, cit., 131. Si veda la Nota del Pontificio Consiglio PER I TESTI LEGISLATIVI, La funzione dell'autorità ecclesiastica sui beni ecclesiastici, 12 febbraio 2004, Communicationes 36 (2004) 24 ss.

${ }^{14}$ Cfr. quanto si riferirà in seguito.

15 Cfr. V. DE PAOLIS, Ricerca di nuovi modelli per gli istituti di vita consacrata nella amministrazione dei loro beni e nella gestione delle opere nella realtà attuale: proposte di soluzione e valutazione, Didaskalia 41 (2011) I, 31 ss. 
tura, all'indole, alla responsabilità e alla deputazione all'apostolato degli istituti. Trattasi di aspetti da salvaguardare e sui quali si è soffermata anche la recente Lettera Circolare della Congregazione per gli istituti di vita consacrata e le società di vita apostolica del 2 agosto 2014, intitolata Linee orientative per la gestione dei beni negli Istituti di vita consacrata e nelle Società di vita apostolica ${ }^{16}$.

Deve infatti sempre tenersi presente il principio fondamentale «che i beni ecclesiastici sono retti dalla legge della Chiesa (can. 1257, \$1) sotto l'autorità dello stesso Sommo Pontefice, in quanto nella Chiesa non può esistere nessuna realtà o autorità che non riconosca il principio dell'unità, che è lo stesso Sommo Pontefice; la Chiesa comunione esiste in forza dell'episcopato, che è l'unità di tutti i vescovi, con Pietro e sotto il capo Pietro» ${ }^{17}$. Pertanto, se la 'fuoriuscita' dei beni dall'ordinamento canonico con l'assegnazione ad un soggetto meramente civilistico costituito $a d$ boc, eludendo così la vigilanza e la supervisione dell'autorità ecclesiastica, è altamente pericolosa, delle insidie presenta pure la permanenza all'interno di questo ordinamento fruendo di un istituto canonistico, forzandone però le norme e avocandosi una competenza dubbia. Invero, del fenomeno della costituzione di enti puramente civili e delle sue gravi controindicazioni quanto ai beni che perdono la qualifica di ecclesiastici e sono 'affrancati' dall'ordinamento canonico si è, con allarme giustificato, da tempo occupata la canonistica, delineando risvolti non poco problematici ${ }^{18}$ : l'opinione di De Paolis è che «se si deve ricorrere all'autorità ecclesiastica per erigere persone giuridiche pubbliche nella Chiesa, a maggior ragione si dovrebbe ricorrere per erigere fondazioni civili, nelle quali i beni temporali non sono più ecclesiastici» ${ }^{19}$; anche nelle pie fondazioni autonome

${ }^{16}$ Cfr. CONGREgazione PER GLI ISTITUTI DI VITA CONSACRATA E LE SOCIETÀ DI VITA APOSTOLICA, Lettera Circolare. Linee orientative per la gestione dei beni negli Istituti di vita consacrata e nelle Società di vita apostolica, 2 agosto 2014, Libreria Editrice Vaticana, Città del Vaticano 2014, spec. 3-6.

${ }^{17}$ V. DE PAOLIS, Ricerca di nuovi modelli..., cit., 38.

${ }^{18}$ Cfr. A. Perlasca, Considerazioni problematiche circa la costituzione di fondazioni civili da parte di istituti religiosi, Informationes SCRIS 27 (2001) passim. Anche O. RODRÍGUEZ MARADIAGA, Il ruolo del vescovo, in giustizia, pace e caritas, Il regno. Documenti 49 (2014) 229, rimarca la necessità di «prestare una particolare attenzione alla situazione degli organismi creati da istituti religiosi che, a volte, non hanno un collegamento istituzionale con coloro dai quali hanno avuto origine».

${ }^{19}$ Cfr. V. DE PaOlis, Ricerca di nuovi modelli..., cit., 63. A tal proposito si veda S. Bueno Salinas, Las personas jurídicas en el derecho canónico, Facultad de Teología de Catalunya, Barcelona 2014, 253: «Tanto en el caso de asociaciones como de fundaciones privadas, los fieles que desean constituirlas en personas jurídicas canónicas han de tener presente que el procedimiento canónico ni supone ni impide el equivalente procedimiento civil, pero que si ambos se llevan a cabo por separado podrán darse en el futuro problemas graves de desajuste (por ejemplo, que una misma asociación aparezca diferentemente constituida ante el Derecho canónico y el Derecho civil, con 
con personalità giuridica privata i beni non sono ecclesiastici, ma restano comunque in qualche modo vincolati a certi controlli ecclesiastici.

In definitiva se «Può esistere la necessità o la opportunità che le opere vengano separate dal patrimonio dell'Istituto, e erette in soggetto giuridico autonomo, particolarmente come fondazione $\gg^{20}$ (soprattutto nel settore sanitario ed educativo), cionondimeno -attesi i suddetti pericoli da ben ponderare- è sommamente apprezzabile che sia la Santa Sede, segnatamente la Congregazione per gli istituti di vita consacrata e le società di vita apostolica, a conferire volta per volta il potere di erezione di una massa bonorum (mobili o immobili) scorporata dall'istituto come fondazione autonoma. Ricorda sempre il cardinale che la Congregazione «quando è favorevole, concede la personalità giuridica canonica pubblica, non privata. In questa infatti i beni temporali non sarebbero più ecclesiastici. Con la costituzione di una pia fondazione autonoma formata dai beni di un settore particolare (come ad esempio, quello sanitario) appartenenti sia ad un singolo istituto, sia a diversi istituti, i beni escono dal patrimonio dell'istituto/istituti e vanno a costituire una nuova persona giuridica pubblica $»^{21}$. Tale intervento apicale non va

diversos estatutos, etc.). Para evitar tal problema, y conociendo que el ordenamiento canónico suele ser más restrictivo que los ordenamientos civiles, tiene más lógica que un ente privado obtenga primero su reconocimiento y erección en el ámbito canónico (pues al cabo se tratará de una asociación o fundación religiosa), y a continuación que resuelva su reconocimiento civil». Si veda inoltre il documento -approvato dal Santo Padre nell'Udienza del 12 dicembre 2017- della Congregazione per gli istituti di vita consacrata e le società di vita apostolica, Economia a servizio del carisma e della missione. Boni dispensatores multiformis gratiae Dei (1 Petr. 4,10). Orientamenti, Libreria Editrice Vaticana, Città del Vaticano 2018. In esso si rileva come vi siano casi nei quali gli istituti di vita consacrata e le società di vita apostolica provvedono alla gestione di alcune opere mediante distinti enti giuridici, spesso organizzati in forma di fondazione o di società (n. 53, ivi, 85): un'opzione ammissibile, fermo quanto stabilito dal diritto universale e proprio, sempre assicurando la fedeltà dell'opera al carisma dell'istituto e la conformità al regime applicabile ai rapporti tra Stato e Chiesa (cfr. n. 85, ivi, 111). Inoltre, la Congregazione attesta come vi siano numerosi enti civili collegati alla persona giuridica canonica (n. 89, ivi, 116-117), in relazione ai quali il diritto proprio deve stabilire le modalità di costituzione e di trasferimento dei beni agli enti stessi. A tal proposito, il documento raccomanda che apposite previsioni negli statuti degli enti civili collegati garantiscano un esercizio del governo in conformità al carisma dell'istituto o della società, per poi puntualizzare come «In nessun caso il ricorso a enti civili, in qualunque forma realizzato, può essere utilizzato per eludere i controlli canonici» (n. 89, ivi, 117).

${ }^{20}$ V. DE PAOLIS, Ricerca di nuovi modelli..., cit., 33.

${ }^{21}$ V. DE PAOLIS, Ricerca di nuovi modelli..., cit., 60, che prosegue in nota: «La Congregazione per gli Istituti di Vita Consacrata sintetizzava così la problematica: "Alcuni istituti religiosi, persuasi che in futuro non saranno più in grado di gestire alcune strutture di cui sono proprietari, al fine di conservare l'identità cattolica delle opere e di proteggere la qualifica di ecclesiastici per i beni che costituiscono il patrimonio di dette opere, hanno chiesto a questo Dicastero la concessione 
percepito come un limite, un ostacolo, un impiccio, ma rappresenta un aiuto nel discernimento, ad esempio sovrintendendo con attenzione la Congregazione (come dimostra la prassi ormai cospicua in atto presso tale dicastero: can. 19) alla redazione degli statuti dell'erigenda fondazione, sì che quanto sopra si è segnalato -specie il rispetto dell'identità ovvero della fisionomia degli istituti religiosi e della finalizzazione dei beni, nonché l'ossequio al magistero della Chiesa in settori cruciali- venga accuratamente presidiato ${ }^{22}$ : al contrario gli «sperimenti isolati di Istituti che percorrono da soli un cammino» ${ }^{23}$ possono rivelarsi deleteri. Il fatto che oggi ci si orienti a fondare pie fondazioni autonome con in dotazione un capitale minimo (e dunque con una minore devoluzione di beni) e con accentuati aspetti organizzativi e di amministrazione non rende tale superiore sorveglianza meno necessaria e conveniente, tutt'altro.

\section{ULTERIORI RAGIONI A CONFORTO DELLA TESI}

Alle ragioni delucidate si possono aggiungere ulteriori considerazioni, tratte da norme codiciali che toccano quadranti correlati a quello ora in esame, nonché dalla speculazione dottrinale in subiecta materia.

\subsection{Il ricorso all'analogia legis. Le autorità competenti ad erigere associazioni pubbliche di fedeli secondo il Codex Iuris Canonici}

Rammentiamo che il Codex Iuris Canonici non specifica l'autorità competente ad erigere persone giuridiche pubbliche o private, ad eccezione delle as-

di persona giuridica pubblica (cfr. cann. 113-123) per l'insieme dei beni del loro apostolato o di una parte di essi. In alcuni casi si tratta di opere di un singolo istituto, che chiede l'erezione di una fondazione autonoma costituita dall'insieme dei beni appartenenti ad un settore (esempio: ospedali) o di diversi istituti, che intendono erigere una fondazione autonoma costituita dall'insieme dei loro beni appartenenti ad un settore del loro apostolato"».

22 «La vigilanza e i controlli non vanno intesi come limitazione dell'autonomia degli enti o segno di mancanza di fiducia, ma come espressione di un servizio alla comunione e alla trasparenza, anche a tutela di chi svolge compiti delicati di amministrazione. La prassi di vigilanza -secondo le modalità determinate dal diritto universale e proprio- non solo risponde al dovere di controllo proprio dei Superiori, ma costituisce un elemento imprescindibile per la natura dei beni ecclesiastici e del loro carattere pubblico, quali mezzi a servizio delle finalità proprie della Chiesa»: CONGREGAZIONE PER GLI ISTITUTI DI VITA CONSACRATA E LE SOCIETÀ DI VITA APOSTOLICA, Lettera Circolare. Linee orientative..., cit., 10.

${ }^{23}$ V. DE PAOLIS, Ricerca di nuovi modelli..., cit., 34. 
sociazioni pubbliche di fedeli. Da questa disciplina si possono trarre spunti per la quaestio ora in esame.

Il can. 312, $\$ 1$ al n. 3 afferma che solo il Vescovo diocesano può erigere associazioni diocesane nell'ambito del suo territorio. Si esclude expressis verbis l'amministratore diocesano, e pure, evidentemente, gli altri ordinari del luogo, anche se hanno potestà di governo esecutiva ordinaria (cfr. anche il can. $134, \$ 3$ ): a maggior ragione non si possono includere gli ordinari ${ }^{24}$. Ciò induce un ragionamento analogico per le fondazioni pie autonome, persone giuridiche alla pari delle universitates personarum. In questo stesso numero la competenza del Vescovo diocesano non si estende alle associazioni per le quali il diritto di erezione è riservato ad altri per privilegio apostolico. Nel $\$ 2$ del medesimo canone si detta che per erigere validamente nella diocesi un'associazione o una sua sezione, anche se ciò avviene in forza di un privilegio apostolico, si richiede il consenso scritto del Vescovo diocesano; tuttavia il consenso del Vescovo diocesano per l'erezione di una casa di un istituto religioso vale anche per l'erezione, presso la stessa casa o presso la chiesa annessa, di una associazione propria di quell'istituto. Come spiega ancora De Paolis, se i Superiori religiosi avessero, in forza del loro ufficio, tale potestà, non avrebbe senso parlare di privilegio apostolico. Facendo il Codice espressa menzione dell'eccezione al potere episcopale nell'erigere persone giuridiche solo in riferimento a istituzioni dotate di un privilegio apostolico, ciò vuol dire che il diritto non prevede altre possibili eccezioni al potere episcopale nell'ambito della diocesi. A livello nazionale e internazionale non sono invece previste alternative rispetto alle autorità indicate nel can. $312, \$ 1,1$ e $2^{25}$. Tale considerazione suffraga per analogia quanto asserito sulle fondazioni pie autonome.

Del resto non pochi canonisti, basandosi proprio sul can. $312, \$ 1$, affermano senza esitazioni in generale: «L'autorità competente per la concessione della personalità giuridica, sia alle persone giuridiche pubbliche sia a quelle private, è di regola, ex can. $312, \S 1$ : -La Santa Sede, per le persone giuridiche di carattere universale e internazionale. -La Conferenza Episcopale, per quelle

${ }^{24}$ Cfr. alcune riflessioni di A. VianA, «Ordinario del lugar», en J. OTADUY - A. VianA - J. SEdANO (eds.), Diccionario General de Derecho Canónico, V, Thomson Reuters Aranzadi, Cizur Menor (Navarra) 2012, 824-825.

25 V. DE PAOLIS, I beni temporali della Chiesa, nuova edizione aggiornata e integrata a cura di A. PERLASCA, Edizioni Dehoniane Bologna, Bologna 2011, 230: in questo volume, nel capitolo VII "Le pie volontà in genere e le pie fondazioni (cann. 1299-1310)", 283 ss., non si fa menzione della questione. 
di carattere nazionale. -Il Vescovo diocesano, per quelle di carattere diocesano ${ }^{26}$. Dunque non sono annoverati i Superiori maggiori degli istituti di diritto pontificio clericali tra le autorità competenti ${ }^{27}$. Ricordiamo infine che per la personalità giuridica delle associazioni private il can. $322, \$ 1$ rinvia al can. 312 .

\subsection{Il criterio ermeneutico del contesto normativo del Titolo IV del Libro $V$}

Nel contesto (can. 17) del Titolo IV («De piis voluntatibus in genere et de piis fundationibus») del Libro V del Codice del 1983, all'ordinarius definito dal can. 134, $\$ 1$ sono conferiti determinati compiti inerenti l'esecuzione, la vigilanza e l'amministrazione delle pie volontà $\mathrm{e}$, in specifico, delle fondazioni pie autonome e non autonome (cfr. cann. 1301, $\$ 1,1304, \$ 1,1305$, $1308, \$ 2$ e 1310, $\$ \$ 1$ e 2), che risulteranno tra l'altro più o meno 'penetranti' se alla fondazione autonoma è stata conferita rispettivamente la personalità giuridica pubblica e privata (can. 116, $₫ 1$ ). Se l'ordinario avesse avuto anche la competenza per l'erezione in persona giuridica essa sarebbe stata esplicitamente nominata (in particolare nel can. 1303).

\subsection{La ratio della qualificazione dei Superiori maggiori de quibus come 'ordinari'}

Un autorevole studioso, premettendo che vi sono delle differenze tra gli ordinari enumerati nel can. 134, $\$ 1$ e, in particolare, evidenziando che i Superiori religiosi «no pertenecen a la organización jerárquica de la Iglesia», in riferimento a questi ultimi rileva: «dentro de los institutos de vida consagrada algunos institutos religiosos y sociedades de vida apostólica necesitan beneficiarse del ejercicio de la potestad de régimen, más allá de la potestad interna que en

${ }^{26}$ L. Chinppetta, Il Codice di Diritto Canonico. Commento giuridico-pastorale, 1, Libri I-II, $3^{\text {a }}$ ed., Edizioni Dehoniane Bologna, Bologna 2011, 144. Spiegando il fatto che il Codex Iuris Canonici, al can. 1303, $\$ 1,1$, non stabilisce a chi compete l'erezione di fondazioni autonome, P. G. MARCUZZI, Le fondazioni pie (cann. 1303-1310 CIC), en I beni temporali della Chiesa, Libreria Editrice Vaticana, Città del Vaticano 1999, 238, conclude: «Il Codice di Diritto Canonico non fa un cenno corrispondente, dal momento che le norme sull'erezione delle persone giuridiche sono state stabilite in modo generale dal Libro I e in modo specifico si trovano poi in altri canoni; di conseguenza, ammette una competenza più ampia per tutte le autorità, che posseggono la potestà di erigere persone giuridiche».

27 Così anche J.-P. SCHOuppe, Elementi di diritto patrimoniale canonico, $2^{\mathrm{a}}$ ed., Giuffrè Editore, Milano 2008, 107: «Sono autorità competenti ad erigere pie fondazioni autonome la Sede Apostolica, la Conferenza episcopale ed il Vescovo diocesano». 
ellos se ejerce en virtud de las constituciones y que tradicionalmente se ha llamado potestad dominativa o asociativa. En efecto, tratándose de institutos y sociedades clericales (cfr. cann. $588 \$ 2$ y $736 \$ 1$ ), los superiores mayores ejercen en esas comunidades la potestad de un ordinario de incardinación, de modo que junto a su función referida a la dirección de los aspectos propios de la vida religiosa en sentido estricto, aquellos superiores velan además por el cumplimiento de los derechos y deberes clericales, al estilo de un obispo para los sacerdotes de su diócesis. Además, tratándose de institutos y sociedades de derecho pontificio (cfr. cann. 589 y 732), es preferible que el obispo diocesano no sea el ordinario ya que de lo contrario resultaría perjudicada la unidad de gobierno de esos institutos y sociedades interdiocesanas. Estas son las causas fundamentales de que algunos superiores religiosos sean ordinarios y otros no. Son las necesidades de la vida religiosa clerical interdiocesana las que reclama esa calificación. [...] el título jurídico que les da la condición de ordinarios no es la exención en cuanto tal, sino el tipo de jurisdicción de que gozan algunos de ellos» ${ }^{28}$. Se così è, e dunque la ratio della normativa va ricondotta alla 'vita religiosa clericale interdiocesana', la qualificazione del Superiore maggiore degli istituti di diritto pontificio clericali come ordinario non pare concernere in alcun modo il conferimento della personalità giuridica alle universitates personarum e alle universitates rerum.

\subsection{Il collegamento tra le persone giuridiche canoniche e la gerarchia ecclesiastica}

Anche altri esperti canonisti, dopo avere sintetizzato i connotati della controversia giuridica di cui ci occupiamo, inclinano nella direzione di non concedere ai Superiori maggiori il potere de quo. In particolare la spiegazione del motivo per cui, fatto salvo un eventuale indulto apostolico (can. $312, \$ 1$, 3) o un mandato speciale, (cfr. can. 134, \$3) l'erezione di persone giuridiche (pubbliche e private) sarebbe riservata alla Santa Sede, alla Conferenza Episcopale oppure al Vescovo diocesano viene ravvisata nella circostanza che le persone giuridiche, in modo particolare quelle pubbliche, anche se il discorso in qualche misura vale anche per quelle private, in quanto agiscono nomine Ecclesiae, cioè ufficialmente, coinvolgono la responsabilità della gerarchia, pur senza tuttavia identificarsi con essa; in ogni caso, si soggiunge, anche gli enti

${ }^{28}$ A. Viana, «Ordinario», en J. OtAdUY - A. Viana - J. Sedano (eds.), DGDC, V, cit., 822-823. 
eretti in forza di un privilegio apostolico (si consideri, a titolo di esempio, un'associazione di fedeli) sottostanno, almeno per qualche aspetto, alla vigilanza del vescovo diocesano (can. $312, \$ 2)^{29}$.

\subsection{La necessaria titolarità della potestas exsecutiva di colui che erige la pia fondazione autonoma}

Altre argomentazioni evidenzia Remigio Beneyto Berenguer, sostenendo risolutamente che sebbene il Codice propriamente non specifichi chi sia il soggetto titolare della potestà necessaria per erigere una pia fondazione autonoma (a differenza del can. 312, $\$ 1$ rispetto alle associazioni pubbliche di fedeli), dato che l'ordinario è l'esecutore di tutte le pie volontà (can. 1301, \$1), che il diritto particolare può e deve determinare condizioni più specifiche per la costituzione delle fondazioni (can. $1304, \$ 2$ ) e che il decreto è un atto amministrativo dell'autorità esecutiva competente (cann. 35, 48 e 135 ss.), pare logico che siano i titolari della potestà esecutiva quelli che possano erigere fondazioni autonome e concedere la personalità giuridica mediante decreto, vale a dire: il Romano Pontefice, i Vescovi diocesani e tutti quelli che sono stati nominati per reggere una Chiesa particolare o una comunità a essa equiparata $(\text { can. } 368)^{30}$.

\subsection{Il confronto comparatistico tra il Codice per la Chiesa latina ed il Codice per le Chiese orientali}

Per risolvere la quaestio iuris può risultare altresì proficuo un confronto sinottico tra il Codex Iuris Canonici del 1983 ed il Codex Canonum Ecclesiarum Orientalium del 1990. Infatti nella codificazione per le Chiese cattoliche orientali, a differenza di quella per la Chiesa latina, il legislatore ha definito quali sono le autorità competenti ad erigere le pie fondazioni autonome: «Piae fundationes autonomae nonnisi ab Episcopo eparchiali aliave auctoritate superiore erigi possunt» $(\text { can. } 1048, \$ 1)^{31}$.

${ }^{29}$ Cfr. A. Perlasca, Considerazioni problematiche..., cit., 85.

${ }^{30}$ Cfr. R. Beneyto Berenguer, «Fundación pía», en J. Otaduy - A. Viana - J. Sedano (eds.), DGDC, IV, Thomson Reuters Aranzadi, Cizur Menor (Navarra) 2012, 167.

31 Cfr. P. G. MarcuzzI, Le fondazioni pie (cann. 1303-1310 CIC), cit., 223 ss., per un'analisi in parallelo della normativa delle due codificazioni (in quella per le Chiese orientali -lo rammentiamo- non è assunta la distinzione tra persone giuridiche pubbliche e private: di conseguenza 
Dal resoconto dell'iter redazionale di tale Codice, inoltre, emerge come i codificatori escludessero espressamente che potessero erigere fondazioni autonome $\mathrm{i}$ «Superiori maggiori dei monasteri maschili e delle Religioni clericali di diritto pontificio» ${ }^{32}$, tanto che alla richiesta di un consultore di ripristinare il can. 300 del Motu Proprio Postquam Apostolicis Litteris di Pio XII (9 febbraio 1952) -ove si stabiliva che $\ll$ Si piae fundationes factae sint in ecclesiis, etiam paroecialibus, monasteriorum virorum cuiusvis condicionis iuridicae aut Religionis clericalis iuris pontificii vel patriarchalis, iura et officia Hierarchae loci, de quibus in cann. 295-299, uni competunt Superiori maiori, firmo can. $65, \S 1,3 »-$ si replicò: «Dopo un approfondito dibattito al riguardo, fatto in due riprese, il $\$ 1$ si lascia immutato, perché esso si riferisce solo alle fundationes autonomae mentre $\mathrm{i}$ diritti dei Superiori maggiori religiosi di cui al can. 300 PA si riferiscono a quelle fondazioni pie che sono definite nel can. 294 PA e che nello schema sono chiamate fundationes non autonomae» ${ }^{33}$. Tali Superiori quindi risultano abilitati solamente, in qualità di Gerarchi (ma non del luogo: can. 984, $\$ 3$ ), ad accettare fondazioni non autonome (can. 1048, $\$ 2$ ).

Osserviamo anche incidentalmente come la redazione del can. 984 del Codex Canonum Ecclesiarum Orientalium sia molto più netta nel sceverare i Superiori maggiori negli istituti di vita consacrata che sono provvisti di potestà di governo ordinaria e che sono gerarchi, dagli altri gerarchi e dai gerarchi del luogo, dedicando ad essi un paragrafo separato del canone, il terzo.

\subsection{L'interpretazione del diritto universale nelle fonti di diritto particolare. L'Istruzione in materia amministrativa della Conferenza Episcopale Italiana}

A riprova della tesi seguita si possono riportare anche le disposizioni della Conferenza Episcopale Italiana, nell'Istruzione in materia amministrativa del $1^{\circ}$ settembre $2005^{34}$.

«tutti i beni delle pie fondazioni autonome [...] sono in ogni caso qualificati come beni ecclesiastici, in conformità al can. $1009 \$ 2$ orientale»: ivi, 229), anche in raffronto alla legislazione precedente ed illustrando i lavori preparatori del diritto vigente.

32 Pontificia Commissio Codici Iuris Canonici Orientalis Recognoscendo, Nuova revisione dello Schema canonum de normis generalibus et de bonis Ecclesiae temporalibus, Nuntia 18 (1984) 68.

33 Pontificia Commissio Codici Iuris Canonici Orientalis Recognoscendo, Nuova revisione..., cit., 68.

34 Cfr. Conferenza Episcopale ITAliana, Istruzione in materia amministrativa, $1^{\circ}$ settembre 2005, Notiziario della Conferenza Episcopale Italiana 8/9 (2005) 329 ss. 
In tale documento pare anzitutto suffragata l'applicazione analogica del can. 312 -che individua nella Sede Apostolica, nella Conferenza Episcopale ${ }^{35}$ e nel Vescovo diocesano le autorità ecclesiastiche competenti a erigere le associazioni di fedeli munite di personalità giuridica pubblica (ma anche privata, posto che, come già riscontrato, al can. 312 rinvia esplicitamente il can. $322, \$ 1$ )- alle ipotesi in cui si debba procedere all'erezione in persona giuridica di una fondazione autonoma pubblica o privata. Infatti il n. 11 così dispone circa l'erezione di persone giuridiche nell'ordinamento canonico: «Il can. 114 non specifica quale sia l'autorità ecclesiastica competente a erigere le persone giuridiche o a conferire a un ente la personalità giuridica. Sovvengono in ogni caso le indicazioni date in altri canoni relativi a enti specifici e la prassi secolare della Chiesa. a) La Santa Sede è competente a erigere persone giuridiche di qualsiasi natura. b) La Conferenza Episcopale è competente a erigere in persona giuridica le associazioni pubbliche di fedeli di rilevanza nazionale (cfr. can. 312 e art. 23, lett. $v$, dello statuto della CEI) e a conferire la personalità giuridica canonica alle associazioni private (cfr. can. $322 \$ 1 \mathrm{e}$ art. 23 , lett. $v$, dello statuto della CEI). c) Il Vescovo diocesano è competente a erigere persone giuridiche di qualsiasi natura nell'ambito della propria giurisdizione, salvo eventuali competenze della Santa Sede (cfr. can. $579 \$ 2$ ). d) I Superiori maggiori degli istituti religiosi di diritto pontificio sono competenti a erigere le province e le case religiose del loro istituto» ${ }^{36}$.

Ma soprattutto, per quanto qui preme, al n. 152 si dichiara recisamente che «L'autorità competente ad erigere in persona giuridica pubblica una pia fondazione autonoma è la Santa Sede o la Conferenza Episcopale o il Vescovo diocesano», estromettendo ex professo dal novero delle autorità competenti i Superiori de quibus. Registriamo tuttavia che stranamente poco prima si era assunto altresì che «Le pie fondazioni autonome sono persone giuridiche pubbliche nell'ordinamento canonico (cfr. cann. $115 \$ \$ 1$ e $3,1303 \$ 1,1^{\circ}$ )», parendo -incongruamente peraltro ${ }^{37}-$ non ammettere pie fondazioni autonome private ${ }^{38}$.

35 Quanto alla Spagna, come vedremo anche in seguito, J. OTADUY, Fundaciones canónicas privadas promovidas por institutos religiosos en los sectores de la educación y de la sanidad, Ius canonicum 55 (2015) 698, riporta il caso di fondazioni di istituti religiosi erette in ambito nazionale dalla Conferenza Episcopale Spagnola «en virtud de las facultades que le confiere el canon $312 \$ 1,2^{\circ}$ del Código de Derecho Canónico».

36 Invero il can. 579 consta di un solo paragrafo.

${ }^{37}$ Cfr. J.-P. SchoupPe, Elementi di diritto patrimoniale canonico, cit., 107.

${ }^{38}$ Cfr. infatti le considerazioni di L. NAVARRo, Le iniziative dei fedeli nel servizio della carità. Fondamento e configurazione giuridica, en J. MiÑAMBRES (ed.), Diritto canonico e servizio della carità, 


\subsection{Le criticità argomentative della tesi positiva. Una lettura non persuasiva e l'esperienza maturata in Spagna}

Le ragioni opposte, a favore cioè della sussistenza del potere in capo ai Superiori maggiori degli istituti di diritto pontificio clericali, che sono apportate dalla dottrina non sono particolarmente convincenti. Invero Francis Morrisey -il quale al termine del suo saggio risalente al 1999 conclude: «The canon 303 associations can be established by those major superiors whom the law recognizes as ordinaries. In other institutes, the diocesan bishop would be the appropriate person to establish such an association (at least at its beginnings)» ${ }^{39}$ - fa leva soprattutto sulla peculiare realtà delle associazioni i cui membri conducono una vita apostolica e tendono alla perfezione cristiana partecipando nel mondo al carisma di un istituto religioso, sotto l'alta direzione dello stesso. L'Autore muove dalla disciplina contenuta nel Codice del 1917 per soffermarsi segnatamente sulla libertà e sul diritto di associazione valorizzato dal Vaticano II e riconosciuto ampiamente dalla codificazione giovanneopaolina, nonché sull'indole e sui caratteri di tali associazioni che condividono lo spirito di istituti religiosi: riflessioni comunque per lo più non estensibili alle pie fondazioni autonome. Desumere poi dai cann. $609, \$ 1$ e 621 che, come il Superiore può erigere case o province dell'istituto religioso (che sono persone giuridiche per il diritto stesso: can. $634, \$ 1$; dunque il decreto del Superiore competente costituisce una comunità religiosa che in forza dell'ordinamento giuridico è persona giuridica pubblica), così il legislatore, pur non avendola prevista, ammette la competenza ad erigere associazioni, non è assolutamente persuasivo. Al contrario ubi lex voluit dixit, ubi noluit tacuit.

Occorrerebbe dunque, semmai, un'apposita riforma de iure condendo che conferisse l'abilitazione specifica dei Superiori maggiori a erigere persone giuridiche pubbliche e private ${ }^{40}$.

Giuffrè Editore, Milano 2008, 193 ss.; IDEM, Diritto dei fedeli e servizio della carità "organizzato", en Pontificio Consiglio Cor Unum (ed.), Il servizio della carità: corresponsabilità e organizzazione, Libreria Editrice Vaticana, Città del Vaticano 2014, 69 ss.

39 F. G. Morrisey, Canon 303 and the establishment of third orders and related association, Informationes SCRIS 25 (1999) 92.

${ }^{40}$ Contra, sembra, F. PANizzolo, La potestà di governo nella vita consacrata. Linee di sviluppo storicogiuridico ed ecclesiologico, Marcianum Press, Venezia 2009, 277, il quale tra i «Doveri specifici dell'Ordinario» inerenti l'esercizio del munus regendi include quello di «conferire personalità giuridica agli insiemi di persone o cose che perseguono un fine effettivamente utile e che siano forniti dei mezzi atti a conseguire il fine stesso che si prefiggono (can. 114, $\$ 3$ )», per poi aggiungere in nota: «Anche questo can. andrebbe collocato nella parte comune a tutti i Superiori maggiori di IR» (ivi, nota 78). 
Quanto alla prassi che si sarebbe instaurata in Spagna, di essa ci informa Jorge Otaduy in un saggio edito nel 2015 ove riferisce che recentemente sono sorte numerose fondazioni «promovidas» ${ }^{41}$ da istituti di vita consacrata principalmente nell'ambito dell'insegnamento (e in quello sanitario). Tali iniziative troverebbero giustificazione, secondo i loro promotori, sia nella gestione altamente professionalizzata esigita dai centri educativi, difficilmente affrontabile da piccole comunità religiose; sia in ragioni economiche, poiché, con la figura della fondazione separata, gli istituti religiosi limitano la loro responsabilità patrimoniale: in caso di fallimento della prima esso non travolgerà i secondi nel naufragio, esonerandoli dal rispondere delle eventuali insolvenze. Secondo tale autorevole canonista, alcune di tali fondazioni sono «constituidas directamente por institutos religiosos que se encuentran capacitados por el Derecho canónico para hacerlo, por disponer de la necesaria potestad eclesiástica de régimen en el fuero externo. Me refiero a los institutos clericales de Derecho pontificio, que pueden actuar a tenor de lo previsto en el canon 596 $\$ 2 \gg^{42}$. Ma una conclusione di tale portata non può essere data per scontata: necessita di alcune precisazioni. Tra l'altro si rileva come in Spagna «El ámbito de actuación de estas fundaciones se extiende al conjunto del territorio nacional o a una parte significativa del mismo, en el que se localizan los centros incorporados a la entidad. En todos los casos se han constituido como fundaciones canónicas privadas» ${ }^{43}$. E tuttavia, in seguito, all'esordio del secondo paragrafo dell'articolo, l'Autore stesso asserisce come negli anni che vanno dal 1992 al 2012 sarebbe stata la Conferenza Episcopale Spagnola ad attribuire alle fondazioni promosse dai religiosi la personalità giuridica di carattere privato $^{44}$, trattando in seguito nel dettaglio sempre esclusivamente di tali fondazioni appunto erette in ambito nazionale dalla Conferenza Episcopale che ne approva gli statuti: ciò che sembrerebbe confermare la tesi che i Superiori degli istituti religiosi sarebbero privi della competenza de qua, solo 'promuovendo' tali fondazioni ${ }^{45}$.

${ }^{41}$ Cfr. J. OTADUY, Fundaciones canónicas privadas..., cit., 696.

42 J. OTADUY, Fundaciones canónicas privadas..., cit., 697.

43 J. OTADUY, Fundaciones canónicas privadas..., cit., 698.

${ }^{44}$ Cfr. J. OTADUY, Fundaciones canónicas privadas..., cit., 702.

45 Cfr. J. OTADUY, Fundaciones canónicas privadas..., cit., 705. Sul tema cfr. anche M. CoRTÉs DiÉGUEZ, Las fundaciones educativas de los institutos de Vida Consagrada. Análisis de sus peculiaridades y de los criterios dados por la Conferencia episcopal para su constitución, Revista española de derecho canónico 72 (2015) 84, la quale rileva che allo scopo di garantire il sostentamento di docenti laici impiegati in istituzioni educative, alcuni istituti di vita consacrata «han solicitado la personalidad 
Non si rinvengono, comunque, argomentazioni teoriche dirimenti a favore di questa presunta prerogativa che si arrogherebbero i Superiori maggiori degli istituti religiosi di diritto pontificio clericali, la quale, pertanto, potrebbe essere reputata abusiva, non rinvenendo fondamento nella normativa codiciale. Del resto vengono enucleate e descritte molte distorsioni ed inconvenienti generati da questa esperienza, sulla quale in definitiva si palesano seri dubbi ${ }^{46}$ : ciò che getta sulla medesima un'ombra la quale non può che confermare quanto si è sostenuto in queste pagine circa la necessaria ed opportuna 'integrazione/sostegno' da parte di altra autorità del potere dei Superiori maggiori degli istituti di diritto pontificio clericali.

\subsection{Le specificazioni desumibili dal Motu Proprio Intima Ecclesiae natura di Benedetto XVI sul servizio della carità nella Chiesa}

Infine, si deve sommare a quanto detto ciò che risulta dalla Lettera Apostolica in forma di Motu Proprio Intima Ecclesiae natura promulgata da Benedetto XVI nel $2012^{47}$ con lo scopo di fornire 'un quadro normativo organi-

jurídica canónica, especialmente de naturaleza privada y con frecuencia de ámbito nacional al amparo de los "Criterios básicos para el régimen de fundaciones canónicas privadas constituidas por institutos de vida consagradas y sociedades de vida apostólica y erigidas por la CEE [Conferenza Episcopale spagnola: N. d. A.]" dados por la Asamblea Plenaria en noviembre 2013». E tali «Criterios básicos» sono stati approvati dalla Conferenza dei Vescovi spagnoli «en virtud de la competencia que le corresponde según sus Estatutos, en consonancia con la disposición del can. 322 del CIC, que le otorga la facultad de conceder personalidad jurídica a las asociaciones de fieles de ámbito nacional» (ivi, 85).

${ }^{46}$ Oltre ai dubbi sulla gestione 'disinvolta' di beni che, mediante vari accorgimenti, finisce per sfuggire ai controlli delle autorità ecclesiastiche (cfr. J. OTADUY, Fundaciones canónicas privadas..., cit., 707 ss.) e a quelli relativi alla garanzia dell'autentica ecclesialità dell'attività svolta in ambiti delicati (cfr. ivi, 714 ss.), ci si sofferma sull'utilizzo strumentale della personalità giuridica privata «para fines estratégicos» (ivi, 719). Infatti tali nuovi soggetti di diritto (pie fondazioni autonome con personalità giuridica privata) non sorgono mediante un atto di autonomia privata dei christifideles, bensì dalla libera iniziativa di una persona giuridica pubblica (l'istituto religioso); inoltre, dall'analisi degli statuti di tali fondazioni emerge come siano soltanto in apparenza ('per finzione') 'private', in quanto permangono alle dirette dipendenze dell'istituto che le ha promosse, figurando perciò sostanzialmente come fondazioni pubbliche; si è dunque desunto che «El caso de estas fundaciones es una manifestación más de la tendencia perceptible en el Derecho canónico reciente, también de ámbito universal, a incrementar el control sobre las personas jurídicas privadas, que daría como resultado una suerte de nuevas entidades tuteladas, como un tertium genus entre públicas y privadas» (ivi, 719).

47 Cfr. Benedetto XVI, Litterae apostolicae Motu Proprio datae Intima Ecclesiae natura «de Caritate Ministranda», 11 novembre 2012, en L'Osservatore Romano, 2 dicembre 2012, 6 ss. (y en AAS 104 [2012] 998 ss.). 
co' per ordinare 'le diverse forme ecclesiali organizzate del servizio della carità' (Proemio) nel contesto della disciplina canonica in vigore ${ }^{48}$, pur potendo tale provvedimento legislativo avere forza derogatoria del diritto codiciale, laddove difforme ${ }^{49}$.

Quali siano le 'forme organizzate' tenute ad ottemperare alle susseguenti disposizioni è indicato all'art. $1^{50}$ il cui $\$ 4$ dispone: 'Gli organismi e le fondazioni promossi con fini di carità dagli Istituti di vita consacrata e Società di vita apostolica sono tenuti all'osservanza delle presenti norme ed in essi deve anche seguirsi quanto stabilito dai cann. $312 \$ 2$ CIC e $575 \$ 2$ CCEO'; «Instituta et opera fundata, caritatis gratia ab Institutis vitae consecratae et Societatibus vitae apostolicae condita, ad has servare normas tenentur atque in iis etiam oportet id servetur quod in cann. $312 \$ 2$ CIC et 575 $\$ 2$ CCEO decernitur».

Il termine latino usato, «conditae», potrebbe tradursi e volere significare 'fondate, istituite, create': ma si deve rilevare che di solito il legislatore, trattando dell'attribuzione della personalità giuridica, usa il vocabolo constituere (cfr. cann. $114, \$ 1$ e $116, \$ 1$ ) ovvero erigere (cfr. cann. $301, \$ \$ 1$ $3,312$, $\$ \$ 1-2$ e $1303, \$ 1,1)$. L'utilizzo delle virgole nel testo ufficiale latino, inoltre, correla immediatamente quel conditae a caritatis gratia, con specifico riferimento, più che al formale atto erettivo, allo scopo al quale le

${ }^{48}$ Infatti come specifica J. I. ARRIETA, Tra responsabilità e servizio. L'espressione canonica del servizio della carità (Commento alla Lettera Apostolica in forma di "Motu Proprio" Intima Ecclesiae natura, dell'11 novembre 2012), en L'Osservatore Romano, 2 dicembre 2012, 9: «Tali disposizioni comuni sono adesso riformulate, assieme ad altre determinazioni provenienti dall'esperienza giuridica e pastorale maturata negli anni, e presentate con una certa organicità per riferimento alle forme ecclesiali organizzate di servizio alla carità».

49 Cfr. H. Pree, Impostazione giuridica del servizio della carità, en Pontificio Consiglio CoR UNUM (ed.), Il servizio della carità: corresponsabilità e organizzazione, cit., 40-41: «Le Litterae Apostolicae Motu Proprio datae de caritate ministranda IEN sono state promulgate su proposta del Cardinale Presidente del Pontificio Consiglio Cor Unum sentito il parere del PCTL, come legge universale per ordine speciale del Papa (cfr. can. $8 \$ 1$ CIC) sul quotidiano "L'Osservatore Romano" e sono entrate in vigore il giorno 10 dicembre 2012. La clausola derogatoria contrariis quibuslibet rebus non obstantibus, etiamsi particulari mentione dignis sottolinea il carattere di una legge speciale della Suprema Autorità e, allo stesso tempo, come legge posteriore ai due Codici, nel caso di contrarietà, la sua forza derogatoria di fronte a questi nonché a qualsiasi altra norma universale o particolare. A causa della funzione complementare dell'IEN relativamente ai Codici, le modificazioni del diritto precedente sono prevalentemente integrazioni».

${ }^{50}$ Come rileva J. I. ARRIETA, Tra responsabilità e servizio..., cit., 9: «L'enunziato normativo probabilmente avrà bisogno d'essere limato nel confronto dottrinale, e risponde a un primo tentativo di identificare un'intera "categoria" di enti che, se intendono agire nel modo determinato dall'articolo 1, per esigenze di giustizia sono tenuti a seguire la disciplina proposta». 
fondazioni sono indirizzate: ed infatti la versione italiana recita 'promossi con fini di carità ${ }^{51}$.

Inoltre una voce assai autorevole -il segretario del Pontificio Consiglio per i testi legislativi- laddove si riferisce alle realtà sorte nell'ambito degli istituti di vita consacrata e delle società di vita apostolica, ha l'accortezza di non adoperare mai l'espressione 'erette da', ma parla di forme «sorte dal lavoro di Istituti di vita consacrata e Società di vita apostolica»: e soprattutto rimarca il collegamento al «ministero di carità affidato ai Pastori», quel «legame essenziale con i Pastori» che pure «deve essere contemperato con un'autonomia proporzionata alle caratteristiche dell'iniziativa ${ }^{52}$. Tra l'altro nelle già evocate Linee orientative per la gestione dei beni negli Istituti di vita consacrata e nelle Società di vita apostolica, la Congregazione per gli istituti di vita consacrata e le società di vita apostolica fa espresso riferimento alle «opere all'interno di ogni

${ }^{51}$ Nella versione in lingua spagnola del Motu Proprio, consultabile all'indirizzo internet www.vatican.va, la frase «Instituta et opera fundata, caritatis gratia ab Institutis vitae consecratae et Societatibus vitae apostolicae condita» è così tradotta: 'Los organismos y las fundaciones que promueven con fines de caridad los Institutos de vida consagrada y Sociedades de vida apostólica'.

52 J. I. ARRIETA, Tra responsabilità e servizio..., cit., 9. Cfr. anche IDEM, Le "Linee orientative per la gestione dei beni negli Istituti di vita consacrata e nelle Società di vita apostolica”, en A. AsTE (ed.), Povertà evangelica, missione e vita consacrata. I beni temporali negli Istituti di vita consacrata e nelle Società di vita apostolica, Marcianum Press, Venezia 2016, 20-21; e F. Catozzella, Una prima lettura del m.p. "Intima Ecclesiae Natura" sul servizio della carità, Apollinaris 86 (2013) 112. A. MonTAN, Vita consacrata e servizio della carità, en PONTIFICIO CONSIGLIO COR UNUM (ed.), Il servizio della carità: corresponsabilità e organizzazione, cit., 93 ss., si sofferma su due importanti affermazioni contenute nell'Intima Ecclesiae natura: «La prima, esplicita e diretta, riguarda il dovere di osservare le norme del Motu Proprio (art. $1 \$ 4$ ) e ciò in forza della natura ecclesiologica degli istituti e delle società, la seconda, implicita ma fondativa, riguarda l'autonomia che deve essere riconosciuta agli istituti e alle società e alle opere caritative ad essi collegate, secondo gli statuti di ciascuna (art. 6), allo scopo di tutelare la loro identità (cann. 578 e $586 \$ 1$ )». La ragione ecclesiologica richiede negli istituti un profondo sensus Ecclesiae, per cui «I servizi di carità, sia propri che quelli collegati, devono risultare permeati, ad un tempo, del genuino spirito della famiglia carismatica a cui appartengono, ma anche di vivo senso ecclesiale mostrando di operare a nome della Chiesa, per suo mandato e nella comunione ecclesiale (can. $675 \$ 3$ ). Il legame con la Chiesa, prima di essere disciplinare, è una questione di appartenenza e deve rispondere alla domanda: quale Chiesa vogliamo essere di fronte alle sfide che quotidianamente ci attendono? Come essere sale della terra, lievito nella pasta, casa sulla roccia, città sul monte, vale a dire come essere Chiesa del Signore? Il servizio di carità, per poter realizzare il principio dell'amore proclamato da Gesù (Gv 15,12-17), deve compiersi mediante una organizzazione conforme alla natura della Chiesa». Anche questo Autore allude al fenomeno, cui sopra abbiamo accennato, che «Ci sono organismi creati da Istituti di vita consacrata e da Società di vita apostolica, dove si distingue tra il riconoscimento civile, perseguito con determinazione, in quanto consente di operare all'interno dello Stato traendo dalla legislazione civile tutti i possibili vantaggi, e il riconoscimento canonico, a volte trascurato o addirittura ignorato» (ivi, 97), esortando al senso di appartenenza alla Chiesa e all'obbligo di conservare sempre la comunione con la medesima. 
Provincia, sia quelle di proprietà dell'Istituto, sia quelle promosse o di emanazione dell'Istituto (esempio associazioni) $\gg^{53}$ (il corsivo è aggiunto): come si supponesse che l'erezione di tali opere (esemplificativamente le universitates personarum, alle quali possono essere aggiunte pure le universitates rerum) non competa direttamente all'Istituto -e, in specie, a colui che ad esso è preposto-, il quale assume un ruolo essenzialmente propulsivo.

È poi possibile evincere un'ulteriore conferma dell'interpretazione approfondita in queste pagine dal richiamo, nell'art. $1, \$ 4$ del Motu Proprio Intima Ecclesiae natura, per tali fondazioni all'osservanza del can. 312, \$2, il quale, come noto, concerne le universitates personarum, segnatamente le associazioni pubbliche di fedeli, e secondo il cui tenore -lo riportiamo ancora- per erigere validamente nella diocesi un'associazione o una sua sezione, anche se ciò avviene in forza di un privilegio apostolico, si richiede il consenso scritto del Vescovo diocesano; tuttavia il consenso dato dal Vescovo diocesano per l'erezione di una casa di un istituto religioso vale anche per l'erezione, presso la stessa casa o presso la chiesa annessa, di una associazione propria di quell'istituto. L'estensione dell'ambito di applicazione del can. 312, $\$ 2$ da parte dell'art. 1, $\$ 4$ dell'Intima Ecclesiae natura determina anzitutto che per erigere nelle diocesi una fondazione promossa con fini di carità si richieda il consenso scritto del Vescovo diocesano; d'altra parte, si tratta verosimilmente di fondazioni che sostengono opere di apostolato esterno dell'istituto religioso, che svolgendosi nel territorio della diocesi sono soggette, oltre che ai Superiori religiosi, altresì alla potestà del Vescovo diocesano (can. 678, $\$ 1)^{54}$. Tale art. 1 , $\$ 4$, nel disporre l'assoggettamento delle fondazioni al can. $312, \S 2$, riconosce che vi sono delle universitates rerum erette in persona giuridica in forza di un privilegio apostolico (parimenti alle universitates personarum). Ciò riconnette inevitabilmente l'art. $1, \$ 4$ dell'Intima Ecclesiae natura al can. 312, $\$ 1,3^{55}$, perché tale privilegio costituisce un'eccezione a una regola generale

53 CONGRegazione Per gli ISTITUTI Di VITA CONSACRATA E LE SOCIETÀ Di VITA APOSTOLICA, Lettera Circolare. Linee orientative..., cit., 11.

${ }^{54}$ Potrebbe anche trattarsi di opere proprie dell'istituto che sono separate o non vincolate rispetto a una casa religiosa anteriormente eretta. In tale caso, a differenza di quanto disposto dal can. 612, «L'attuale Codice di Diritto Canonico non ha alcuna disposizione che tratti specificatamente questo punto», anche se in dottrina il consenso previo del Vescovo diocesano è comunque ritenuto necessario: cfr. P. H. KolvENBACH, Relazioni tra Vescovi e Superiori generali nelle questioni riguardanti fondazioni e chiusura di opere, Informationes SCRIS 24 (1998) 53-54.

55 Sulla connessione tra il can. $312, \$ 1,3$ e il $\$ 2$ del canone medesimo, relativamente agli istituti religiosi cfr. V. DE PAOLIS, I beni temporali della Chiesa, cit., 330. 
per cui solamente il Vescovo diocesano (can. 134, \$3) può erigere nella diocesi fondazioni autonome con personalità giuridica pubblica ovvero privata (can. 322, \$ 1). Il Motu Proprio di Papa Benedetto XVI ha dunque reso esplicita la riserva del Vescovo diocesano derivante dall'applicazione analogica del can. $312, \$ 1$ circa l'erezione di fondazioni autonome di cui al can. $1303, \$ 1,1$, che perciò non compete ai Superiori maggiori degli istituti religiosi di diritto pontificio clericali, anche se ordinari ai sensi del can. $134, \$ 1$ e titolari della potestà ecclesiastica di governo a norma del can. 596, $\$ 2$, a meno che non agiscano in forza di un privilegio apostolico: in questo caso possono erigere una fondazione autonoma pubblica o privata, ma pur sempre osservando l'art. 1, $\$ 4$ dell'Intima Ecclesiae natura, dovendo richiedere ed ottenere il previo consenso del Vescovo diocesano.

Può inoltre invocarsi, quanto al regimen, l'art. 3 del Motu Proprio del 2012, secondo il cui tenore ' $\$ 1$ Agli effetti degli articoli precedenti, s'intende per autorità competente, nei rispettivi livelli, quella indicata dai cann. 312 CIC e 575 CCEO. $\$ 2$ Trattandosi di organismi non approvati a livello nazionale, anche se operanti in varie diocesi, per autorità competente si intende il Vescovo diocesano del luogo dove l'ente abbia la sua sede principale. In ogni caso, l'organizzazione ha il dovere di informare i Vescovi delle altre diocesi ove operasse, e di rispettare le loro indicazioni riguardanti le attività delle varie entità caritative presenti in diocesi'. Come nota Pree, «In generale, IEN non muta le competenze previste nel CIC/CCEO relative alla disciplina delle associazioni e delle fondazioni, sebbene comporti notevoli precisazioni ed integrazioni nei campi della vigilanza nonché delle pie fondazioni. È degno di nota che la regola generale sulle autorità competenti nell'art. $3 \$ 1$ IEN dev'essere applicata tanto alle associazioni quanto alle fondazioni con fine di carità; questo risulta dal rinvio agli art. precedenti, i quali comprendono tutt'e due i tipi di enti» ${ }^{56}$. Sempre commentando tale articolo, lo stesso Autore indugia sul ruolo del Vescovo diocesano ${ }^{57}$, ribadendo la 'sottomissio-

${ }^{56}$ H. PREE, Impostazione giuridica del servizio della carità, cit., 49.

${ }^{57}$ Cfr. H. PrEe, Impostazione giuridica del servizio della carità, cit., 51: «Rinviando ai can. 312 CIC rispettivamente can. 575 CCEO, l'art. $3 \$ 1 \mathrm{IEN}$ stabilisce come autorità competente a livello diocesano/eparchiale il Vescovo diocesano/eparchiale. L'espressione "Vescovo diocesano/eparchiale" è da intendersi ai sensi del can. $134 \$ 3$ CIC / can. 987 CCEO, come risulta dall'art. 4 $\$ 1$ IEN. Ne consegue che, in quanto si tratta di atti amministrativi, la competenza spetta soltanto al Vescovo e agli altri a lui stesso equiparati in diritto, esclusi il Vicario generale ed Episcopale (Protosincello e Sincello), se non per mandatum speciale. È degno di nota che l'IEN attribuisce quasi tutte le competenze relative al servizio della carità al Vescovo diocesano/eparchiale, 
ne alla potestà' di quest'ultimo degli enti con fini di carità, siano associazioni oppure fondazioni, promossi da istituti di vita consacrata o società di vita apostolica $^{58}$.

\subsection{La potestà dei Superiori maggiori in relazione alla costituzione di universitates rerum nella riflessione dottrinale}

Dalla lettura dei contributi di canonisti che si sono cimentati con esposizioni generali circa la materia delle pie fondazioni autonome nel diritto della Chiesa, risulta come pressoché nessuno abbia sostenuto ex profess ${ }^{59}$ che i Superiori maggiori degli istituti di diritto pontificio clericali siano muniti della potestà di erigere universitatem rerum con personalità giuridica pubblica o privata.

A conferma -oltre a quelli che abbiamo avuto modo di citare- alleghiamo un breve elenco di Autori da noi consultati che accennano, è vero, al problema, ma paiono inclinare per la soluzione contraria alla titolarità del potere dei Superiori religiosi, ovvero non si pronunciano decisamente per la revisione dell'anteriore normativa, lasciando dunque intendere che il regime è rimasto identico a quello sussistente sotto l'imperio della codificazione pianobenedettina.

mai all'Ordinario/Gerarca, eccetto unicamente art. $10 \$ 5$ (rendiconto annuale da presentare all'Ordinario). Questo sottolinea il peso che il legislatore conferisce alla diakonia rispettivamente alla responsabilità del Vescovo al riguardo».

${ }^{58}$ Cfr. H. PREE, Impostazione giuridica del servizio della carità, cit., 54: «Enti con fini di carità, siano associazioni oppure fondazioni, promossi da IVC o SVA sono tenuti all'osservanza delle norme dell'IEN nonché di quanto è stabilito dai can. $312 \$ 2$ CIC rispettivamente can. $575 \$ 2$ CCEO. Tutti gli enti caritativi degli IVC e SVA, anche di Diritto pontificio, sono sottomessi alla potestà del Vescovo diocesano/eparchiale, rispettivamente alla Conferenza episcopale o al PC Cor Unum (cfr. art. $3, \$ 1 \mathrm{IEN)}$. Questo è conforme a quanto è stabilito dal can. $678 \$ 1$ rispettivamente dai cann. $415 \$ 1$ e $554 \$ 2$ CCEO: tutti i religiosi sono soggetti alla potestà del Vescovo / Gerarca del luogo nelle cose che riguardano, fra le altre, le opere dell'apostolato (in senso largo, compreso il servizio della carità)». Pree, peraltro, si interroga: «Si pone la questione se le norme dell'art. $10 \$ \$ 1$ e 5 IEN, vuol dire sulla vigilanza del Vescovo sui beni ecclesiastici degli organismi caritativi "soggetti alla sua autorità" nonché sul rendiconto annuale, devono essere applicate agli enti promossi da IVC o SVA indipendentemente da se si tratta di un Istituto di diritto pontificio oppure di diritto diocesano, perché gli Istituti godono della giusta autonomia (can. 586 CIC) e l'amministrazione dei beni rientra nella sfera di detta autonomia. Anche queste norme necessitano una precisazione in quanto all'ambito d'applicazione, p. es.: Cosa significa "soggetti alla sua autorità" relativamente a IVC e SVA in questo contesto? Sono i beni ecclesiastici, di cui parla il $\$ 1$, quelli secondo can. $1057 \$ 1$ CIC oppure anche i beni di enti caritativi che non sono personae iuridicae publicae?» (ivi, 54-55).

59 Cfr. peraltro l'opinione di F. PANIZZOLO, La potestà di governo..., cit., che abbiamo sopra riportato. 
Così, trattando del riconoscimento della personalità giuridica, Gaetano Lo Castro non enumera tra le autorità competenti i Superiori maggiori degli istituti di diritto pontificio clericali ${ }^{60}$.

José María Vázquez García-Peñuela non menziona in alcun punto della sua esposizione le pie fondazioni autonome correlate agli istituti religiosi. Sulle autorità competenti all'erezione non manifesta la sua opinione riportando quella altrui: «La erección corresponde, a tenor del $\$ 1$, a "la autoridad eclesiástica competente". Según Traserra, y aun opinando que por razones prácticas hubiera sido mejor reservar la materia al Obispo diocesano, debe comprenderse bajo esa expresión "también a los Vicarios generales y episcopales, en cuanto titulares de potestad ejecutiva ordinaria (can. $134 \$ 1$ ), e incluso a los que hubiesen obtenido delegación de los titulares de la potestad ejecutiva ordinaria (can. 137)" $\gg^{61}$.

Altre opere monografiche ${ }^{62}$ e collettanee ${ }^{63}$ ignorano la disputa, mentre Antonio Calabrese, presumibilmente limitandosi all'analisi dei canoni sulle pie fondazioni che richiamano esplicitamente l'ordinario di cui al can. 134, $\$ 1$, annota: «L'ordinario competente in tutta questa materia, fatta eccezione per quanto riguarda la durata delle pie fondazioni per la quale è competente sempre l'ordinario del luogo, è quello proprio, che per gli istituti clericali di diritto pontificio è il superiore maggiore, il quale nel diritto è chiamato anche ordinario, ma per gli altri istituti è l'ordinario del luogo» ${ }^{64}$.

\section{ApProdo CONCLUSIVO. L'UTILITÀ DI APPRONTARE UNA MISURA CHIARIFICATRICE PER VIA LEGISLATIVA}

Alla luce di quanto sinora illustrato riteniamo si possa giungere alla conclusione che i Superiori maggiori degli istituti di diritto pontificio clericali

${ }^{60}$ Cfr. G. Lo Castro, sub can. 114, en Á. Marzoa - J. Miras - R. RodríGuez-Ocaña (eds.), Comentario exegético al Código de Derecho Canónico, I, $3^{\text {a }}$ ed., Eunsa, Pamplona 2002, 782 ss.

${ }^{61}$ J. M. VÁzQUez García-Peñulla, sub can. 1303, en Á. MarzoA - J. Miras - R. RodríguezOCaÑa (eds.), Comentario exegético al Código de Derecho Canónico, IV/1, $3^{\mathrm{a}}$ ed., Eunsa, Pamplona 2002, 197 [per il rimando cfr. J. TraserRa CunILlERA, Las fundaciones pías autónomas, Facultad de Teología de Barcelona, Barcelona 1985, 40].

62 Cfr. C. Begus, Diritto patrimoniale canonico, Lateran University Press, Città del Vaticano 2007, 135 ss., nel capitolo incentrato su "Le pie fondazioni".

${ }^{63}$ Cfr. V. Mosca (ed.), Vita consacrata e gestione delle opere, Libreria Editrice Vaticana, Città del Vaticano 2014, $131 \mathrm{pp}$.

${ }^{64}$ A. CALABRESE, Istituti di vita consacrata e società di vita apostolica, $3^{\mathrm{a}}$ ed., Libreria Editrice Vaticana, Città del Vaticano 2011, 162. 
non siano titolari della potestà esecutiva per erigere pie fondazioni autonome con personalità giuridica pubblica o privata, in quanto sussiste una riserva, nel novero dei soggetti indicati dal can. $312, \$ 1$ (a seconda dell'ambito di operatività della fondazione), a favore della Sede Apostolica, poiché gli istituti di diritto pontificio sono soggetti in modo immediato ed esclusivo alla sua potestà (can. 593): i Superiori potrebbero erigere una fondazione agendo in forza di un privilegio apostolico ${ }^{65}$ ovvero, così come opina De Paolis, in forza di una norma apposita inclusa nelle loro Costituzioni approvate dalla Santa Sede ${ }^{66}$. L'inabilità nei casi ordinari dei Superiori religiosi vale sia per le fondazioni di rilevanza esterna sia per quelle di rilevanza interna all'istituto, anche se in quest'ultimo caso appare logico, più che creare un nuovo ente con personalità giuridica pubblica o privata, che i Superiori si avvalgano della normativa sulla fondazione non autonoma di cui al can. $1303, \$ 1,2^{67}$ : fondazioni che essi possono validamente accettare in quanto ordinari, assicurando inoltre prudenzialmente che i beni siano devoluti a una persona giuridica pubblica (l'istituto religioso) e, perciò, acquisiscano lo status giuridico di bene ecclesiastico (can. 1257, \$1).

In ogni caso, posto che si tratta di una questione di diritto non semplice che investe indirettamente altri snodi cruciali circa il diritto dei religiosi-come la natura della potestà di governo dei Superiori degli istituti religiosi di diritto pontificio clericali, le ripercussioni giuridico-ecclesiologiche della qualificazione di tali Superiori come 'ordinari', i rapporti tra Superiori religiosi e Congregazione romana, le interrelazioni tra Superiori religiosi e Vescovi diocesani nell'ambito della Chiesa particolare, l'amministrazione dei beni degli istituti religiosi mediante l'erezione di fondazioni autonome anche private- non può escludersi l'opportunità di promulgare un'interpretazione autentica per modum

${ }^{65}$ In questo caso, tuttavia, se si tratta di una fondazione promossa con fini di carità è necessario ottenere il consenso scritto del Vescovo diocesano ai sensi dell'art. $1, \$ 4$ del Motu Proprio Intima Ecclesiae natura e del can. $312, \$ 2$. E comunque, al di là del Motu Proprio di Benedetto XVI, un qualche intervento 'confermativo' dell'autorità episcopale competente andrebbe richiesto in ogni caso, anche per fondazioni non destinate a finanziare opere di carità, specialmente per quanto concerne le opere di apostolato esterno all'istituto.

${ }^{66}$ Occorrerebbe tuttavia analizzare la portata di questa approbatio: se fosse considerata di natura meramente amministrativa, opererebbe l'art. 18 della Costituzione Apostolica Pastor Bonus, per cui i dicasteri non potrebbero derogare alla legge... De iure condito è più prudente concludere che la Congregazione dei religiosi può solo concedere privilegi apostolici di tipo singolare, non potendo approvare norme costituzionali che, di fatto, derogherebbero alla disciplina codiciale.

${ }^{67}$ Cfr. alcune considerazioni di S. Bueno Salinas, «Persona jurídica», en J. OTADUY - A. VIANA J. SEDANo (eds.), DGDC, VI, Thomson Reuters Aranzadi, Cizur Menor (Navarra) 2012, 182. 
legis del can. 1303, $\$ 1,1$, che, là dove sia esplicativa di una legge oggettivamente dubbia (can. 16, $\$ 2$ ), implicherebbe l'applicazione del principio secondo cui lex dubia non obligat (can. 14) ${ }^{68}$. In base a tale principio, nel caso in cui l'interpretazione autentica del Pontificio Consiglio per i testi legislativi ${ }^{69}$ (art. 155 della Costituzione Apostolica Pastor bonus) rispondesse negativamente al quesito se i Superiori de quibus, in quanto ordinari, possano erigere pie fondazioni autonome pubbliche o private, non sarebbe travolta la validità degli eventuali atti istitutivi delle fondazioni autonome pubbliche o private posti eventualmente in essere dai Superiori maggiori stessi in precedenza.

${ }^{68}$ Sull'interpretazione autentica esplicativa cfr. M. GANARIN, L'interpretazione autentica nelle attuali dinamiche evolutive del diritto canonico, Bonomia University Press, Bologna 2018, 127 ss., e i riferimenti bibliografici ivi riportati.

${ }^{69}$ Cfr. R. J. Castillo Lara, De iuris canonici authentica interpretatione in actuositate Pontificiae Commissionis adimplenda, Communicationes 20 (1988) 265 ss.; F. J. URrutia, De Pontificio Consilio de Legum Textibus Interpretandis, Periodica de re morali canonica liturgica 78 (1989) 503 ss.; V. FAGIOLO, Competenza e struttura del dicastero per la funzione interpretativa delle leggi della Chiesa, Nomos. Le attualità del diritto 4 (1991) 7 ss.; J. HeRranz, L'interpretazione autentica: il Pontificio Consiglio per l'Interpretazione dei Testi Legislativi, en Associazione CanONISTICA ITALiana (ed.), Il diritto della Chiesa. Interpretazione e prassi, Libreria Editrice Vaticana, Città del Vaticano 1996, 65 ss.; G. INCITTI, L'interpretazione e il Pontificio Consiglio per [l'Interpretazione dei] i Testi Legislativi, en Gruppo Italiano Docenti di Diritto Canonico (ed.), Fondazione del diritto. Tipologia e interpretazione della norma canonica, Glossa, Milano 2001, 153 ss.; T. BERTONE, La legge canonica e il governo pastorale della Chiesa: il ruolo specifico del Pontificio Consiglio per i Testi Legislativi, en Pontificio Consiglio Per I testi legislativi (ed.), La legge canonica nella vita della Chiesa. Indagine e prospettive nel segno del recente magistero pontificio, Libreria Editrice Vaticana, Città del Vaticano 2008, 29 ss.; F. CocCopalmerio, Il Pontificio Consiglio per i Testi Legislativi, en L. SABBARESE (ed.), La Chiesa è missionaria. La ricezione nel Codice di Diritto Canonico, Urbaniana University Press, Città del Vaticano 2009, 205 ss.; J. I. ARRIETA, Evoluzione del Pontificio Consiglio per $i$ Testi Legislativi dopo la promulgazione della Cost. Ap. Pastor Bonus, Ephemerides iuris canonici 50 (2010) 121 ss.; M. GANARIN, Il Pontificio Consiglio per i Testi Legislativi nell'assetto istituzionale della Curia romana tra diritto vigente e prospettive di riforma, Stato, Chiese e pluralismo confessionale, Rivista telematica (www.statoechiese.it), n. 22/2015, 22 giugno 2015, 1 ss. 
IN MERITO AL PROBLEMA SE I SUPERIORI MAGGIORI DEGLI ISTITUTI RELIGIOSI

\section{Fonti}

BenedetTo XVI, Litterae apostolicae Motu Proprio datae Intima Ecclesiae natura «de Caritate Ministranda», 11 novembre 2012, en L'Osservatore Romano, 2 dicembre 2012, 6-7 (y en AAS 104 [2012] 996-1004).

CONFERENZA EPISCOPALE ITALIANA, Istruzione in materia amministrativa, $1^{\circ}$ settembre 2005, Notiziario della Conferenza Episcopale Italiana 8/9 (2005) $329-422$.

CONGREGAZIONE PER GLI ISTITUTI DI VITA CONSACRATA E LE SOCIETÀ DI VITA APOSTOLICA, Lettera Circolare. Linee orientative per la gestione dei beni negli Istituti di vita consacrata e nelle Società di vita apostolica, 2 agosto 2014, Libreria Editrice Vaticana, Città del Vaticano 2014.

Pontificia Commissio Codici Iuris Canonici Orientalis RecognoscenDO, Nuova revisione dello Schema canonum de normis generalibus et de bonis Ecclesiae temporalibus, Nuntia 18 (1984) 68.

Pontificia Commissio Codici Iuris Canonici Recognoscendo. Coetus STUDIORUM «DE BONIS ECCLESIAE TEMPORALIBUS», Sessio II (diebus 12-16 novembris 1979 babita). Adunatio diei 15 novembris 1979, Communicationes 12 (1980) 435.

PONTIFICIO CONSIGLIO PER I TESTI LEGISLATIVI, La funzione dell'autorità ecclesiastica sui beni ecclesiastici, 12 febbraio 2004, Communicationes 36 (2004) 24-32.

\section{Bibliografía}

ANDRÉs, D., Le forme di vita consacrata. Commentario teologico-giuridico al Codice di Diritto Canonico, 6a ed., Ediurcla, Roma 2008.

—, Los Superiores religiosos de los religiosos según el Código: IV Estatuto específico de los Superiores mayores Ordinarios, Commentarium pro religiosis et missionariis 79 (1998) 159-191.

ARRIETA, J. I., Le "Linee orientative per la gestione dei beni negli Istituti di vita consacrata e nelle Società di vita apostolica”, en A. Aste (ed.), Povertà evangelica, missione e vita consacrata. I beni temporali negli Istituti di vita consacrata e nelle Società di vita apostolica, Marcianum Press, Venezia 2016, 11-28.

—, Tra responsabilità e servizio. L'espressione canonica del servizio della carità (Commento alla Lettera Apostolica in forma di "Motu Proprio" Intima Ecclesiae natura, dell'11 novembre 2012), en L'Osservatore Romano, 2 dicembre 2012, 9.

—, Evoluzione del Pontificio Consiglio per i Testi Legislativi dopo la promulgazione della Cost. Ap. Pastor Bonus, Ephemerides iuris canonici 50 (2010) 121-133. 
Begus, G., Diritto patrimoniale canonico, Lateran University Press, Città del Vaticano 2007.

Beneyto Berenguer, R., «Fundación pía», en J. Otaduy - A. Viana - J. SEDANO (eds.), Diccionario General de Derecho Canónico, IV, Thomson Reuters Aranzadi, Cizur Menor (Navarra) 2012, 163-170.

Bertone, T., La legge canonica e il governo pastorale della Chiesa: il ruolo specifico del Pontificio Consiglio per $i$ Testi Legislativi, en PONTIFICIO CONSIGLIO PER I TESTI LEGISLATIVI (ed.), La legge canonica nella vita della Chiesa. Indagine e prospettive nel segno del recente magistero pontificio, Libreria Editrice Vaticana, Città del Vaticano 2008, 29-43.

Bueno Salinas, S., Las personas jurídicas en el derecho canónico, Facultad de Teología de Catalunya, Barcelona 2014.

—, «Persona jurídica», en J. Otaduy - A. Viana - J. SEdano (eds.), Diccionario general de derecho canónico, VI, Thomson Reuters Aranzadi, Cizur Menor (Navarra) 2012, 179-188.

Calabrese, A., Istituti di vita consacrata e società di vita apostolica, $3^{\mathrm{a}}$ ed., Libreria Editrice Vaticana, Città del Vaticano 2011.

Castillo LaRa, R. J., De iuris canonici authentica interpretatione in actuositate Pontificiae Commissionis adimplenda, Communicationes 20 (1988) 265-287.

Catozzella, F., Una prima lettura del m.p. "Intima Ecclesiae Natura" sul servizio della carità, Apollinaris 86 (2013) 99-123.

Chiappetta, L., Il Codice di Diritto Canonico. Commento giuridico-pastorale, 1, Libri I-II, $3^{a}$ ed., Edizioni Dehoniane Bologna, Bologna 2011.

Coccopalmerio, F., Il Pontificio Consiglio per $i$ Testi Legislativi, en L. SabbareSE (ed.), La Chiesa è missionaria. La ricezione nel Codice di Diritto Canonico, Urbaniana University Press, Città del Vaticano 2009, 205-222.

CorTés Diéguez, M., Las fundaciones educativas de los institutos de Vida Consagrada. Análisis de sus peculiaridades y de los criterios dados por la Conferencia episcopal para su constitución, Revista española de derecho canónico 72 (2015) 73-94.

DE PAolis, V., I beni temporali della Chiesa, nuova edizione aggiornata e integrata a cura di A. PERLASCA, Edizioni Dehoniane Bologna, Bologna 2011.

-, Ricerca di nuovi modelli per gli istituti di vita consacrata nella amministrazione dei loro beni e nella gestione delle opere nella realtà attuale: proposte di soluzione e valutazione, Didaskalia 41 (2011) I, 31-65.

-, La vita consacrata nella Chiesa, edizione rivista e ampliata a cura di V. MosCA, Marcianum Press, Venezia 2010.

—, L'autorità competente ad erigere una persona giuridica nella Chiesa, Periodica de re canonica 92 (2003) 3-20 e 223-255. 
—, L'autorità competente ad erigere una persona giuridica nella Chiesa, Informationes SCRIS 26 (2000) 59-86.

EsPosito, B., Alcune riflessioni sul Superiore maggiore in quanto Ordinario e sulla valenza ecclesiologica e canonica della qualifica, Angelicum 78 (2001) 669-731.

Fagiolo, V., Competenza e struttura del dicastero per la funzione interpretativa delle leggi della Chiesa, Nomos. Le attualità del diritto 4 (1991) 7-19.

GANARIN, M., L'interpretazione autentica nelle attuali dinamiche evolutive del diritto canonico, Bonomia University Press, Bologna 2018.

—, Il Pontificio Consiglio per i Testi Legislativi nell'assetto istituzionale della Curia romana tra diritto vigente e prospettive di riforma, Stato, Chiese e pluralismo confessionale, Rivista telematica (www.statoechiese.it), n. 22/2015, 22 giugno 2015, 1 ss.

Herranz, J., L'interpretazione autentica: il Pontificio Consiglio per l'Interpretazione dei Testi Legislativi, en Associazione Canonistica Italiana (ed.), Il diritto della Chiesa. Interpretazione e prassi, Libreria Editrice Vaticana, Città del Vaticano 1996, 65-82.

InCITTI, G., Linterpretazione e il Pontificio Consiglio per [l'Interpretazione dei] $i$ Testi Legislativi, en Gruppo Italiano Docenti di DiritTo Canonico (ed.), Fondazione del diritto. Tipologia e interpretazione della norma canonica, Glossa, Milano 2001, 153-178.

KolvenBaCH, P. H., Relazioni tra Vescovi e Superiori generali nelle questioni riguardanti fondazioni e chiusura di opere, Informationes SCRIS 24 (1998) 49-56.

Lo Castro, G., sub can. 114, en Á. MarzoA - J. Miras - R. RodríguezOCaña (eds.), Comentario exegético al Código de Derecho Canónico, I, $3^{\mathrm{a}}$ ed., Eunsa, Pamplona 2002, 777-786.

López Alarcón, M., sub can. 1303, en J. I. Arrieta (ed.), Codice di diritto canonico e leggi complementari commentato, $5^{\text {a }}$ ed., Coletti a San Pietro, Roma 2015, 868-869.

MARCUZZI, P. G., Le fondazioni pie (cann. 1303-1310 CIC), en I beni temporali della Chiesa, Libreria Editrice Vaticana, Città del Vaticano 1999, 223-259.

MiÑambres, J., Fondazioni pie e figure affini, Ius Ecclesiae 21 (2009) 333-345.

Montan, A., Vita consacrata e servizio della carità, en Pontificio Consiglio COR UNUM (ed.), Il servizio della carità: corresponsabilità e organizzazione, Libreria Editrice Vaticana, Città del Vaticano, 2014, 92-102.

Morrisey, F. G., Canon 303 and the establishment of third orders and related association, Informationes SCRIS 25 (1999) 74-92. 
Mosca, V. (ed.), Vita consacrata e gestione delle opere, Libreria Editrice Vaticana, Città del Vaticano 2014.

Navarro, L., Diritto dei fedeli e servizio della carità "organizzato", en PONTIFICIO CONSIGLIO COR UNUM (ed.), Il servizio della carità: corresponsabilità e organizzazione, Libreria Editrice Vaticana, Città del Vaticano 2014, 69-91.

—, Le iniziative dei fedeli nel servizio della carità. Fondamento e configurazione giuridica, en J. MiÑAmbres (ed.), Diritto canonico e servizio della carità, Giuffrè Editore, Milano 2008, 193-224.

OTADUY, J., Fundaciones canónicas privadas promovidas por institutos religiosos en los sectores de la educación y de la sanidad, Ius canonicum 55 (2015) 695-722.

PANIZZOLO, F., La potestà di governo nella vita consacrata. Linee di sviluppo storico-giuridico ed ecclesiologico, Marcianum Press, Venezia 2009.

PerLasCa, A., Considerazioni problematiche circa la costituzione di fondazioni civili da parte di istituti religiosi, Informationes SCRIS 27 (2001) 75-90.

PREE, H., Impostazione giuridica del servizio della carità, en PONTIFICIO CONSIGLIO COR UNUM (ed.), Il servizio della carità: corresponsabilità e organizzazione, Libreria Editrice Vaticana, Città del Vaticano 2014, 39-68.

RinCón-PÉREZ, T., sub can. 596, en Á. MARZOA - J. Miras - R. RodríGUEZOCAÑa (eds.), Comentario exegético al Código de Derecho Canónico, II/2, $3^{\mathrm{a}}$ ed., Eunsa, Pamplona 2002, 1473-1479.

Rodríguez Maradiaga, O., Il ruolo del vescovo, in giustizia, pace e caritas, Il regno. Documenti 49 (2014) 225.

Schouppe, J.-P., Elementi di diritto patrimoniale canonico, $2^{\mathrm{a}}$ ed., Giuffrè Editore, Milano 2008.

Traserra Cunillera, J., Las fundaciones pías autónomas, Facultad de Teología de Barcelona, Barcelona 1985.

Urrutia, F. J., De Pontificio Consilio de Legum Textibus Interpretandis, Periodica de re morali canonica liturgica 78 (1989) 503-546.

VÁzquez García-Peñuela, J. M., sub can. 1303, en Á. MarzoA - J. Miras R. Rodríguez-Ocaña (eds.), Comentario exegético al Código de Derecho Canónico, IV/1, 3ª ed., Eunsa, Pamplona 2002, 193-203.

Viana, A., «Ordinario», en J. Otaduy - A. Viana - J. Sedano (eds.), Diccionario General de Derecho Canónico, V, Thomson Reuters Aranzadi, Cizur Menor (Navarra) 2012, 819-823.

—, «Ordinario del lugar», en J. Otaduy - A. Viana - J. Sedano (eds.), Diccionario General de Derecho Canónico, V, Thomson Reuters Aranzadi, Cizur Menor (Navarra) 2012, 823-825. 


\section{INFORMACIÓN E INSTRUCCIONES PARA LOS AUTORES Y REVISORES}

1. La revista Ius Canonicum publica dos números por año, en junio y diciembre. El contenido del primer número del año queda definitivamente fijado el 30 de marzo, mientras que el segundo es cerrado el 30 de septiembre; sin perjuicio, naturalmente, de que la programación de la Revista alcance más allá del año en curso.

2. Los autores someterán sus artículos a través de la plataforma OJS (Open Journal Systems), adaptada para Ius Canonicum, a través del siguiente enlace: http://www. unav.edu/publicaciones/revistas/index.php/ius-canonicum/.

Durante todo el proceso de preparación del número de la revista, OJS será el cauce de comunicación entre el autor y el editor. Para resolver cualquier duda en relación con el procedimiento los autores pueden dirigirse a: rsjimenez@unav.es.

3. Las colaboraciones deberán ser textos inéditos y originales. El autor podrá usar libremente el texto cuando haya sido publicado, con la oportuna cita de Ius Canonicum como lugar original de publicación. La Revista juzgará la posibilidad de publicar en ocasiones la versión española de textos presentados en Congresos y aún no publicados; en tal caso se hará constar esta circunstancia en una nota previa a pie de página.

4. Las colaboraciones para las secciones de «estudios» y de «comentarios» no deberán superar las 15.000 palabras, incluidas las notas a pie de página. Constará el nombre y apellidos del autor, así como el cargo académico o profesional (solamente uno) y la dirección del correo electrónico. Además, se incluirá al final del artículo la relación completa de la bibliografía citada y ordenada alfabéticamente por apellido (la inicial del nombre se coloca a continuación del apellido).

5. Los textos que sean destinados a la sección de «estudios» deberán contener un resumen de 150 palabras en español y en inglés. Igualmente, se incluirán en ambas lenguas, tres palabras clave (keywords).

6. Una vez recibido el original, la Revista informará al autor del comienzo del proceso anónimo de revisión. Éste consiste en la evaluación del texto por parte de dos revisores externos al Consejo Editorial de la Revista, que juzgan si su forma y contenido se ajustan a los criterios científicos vigentes en el derecho canónico y disciplinas afines. El Director de la Revista comunicará al autor el resultado del proceso revisor, indicando si el escrito ha sido aceptado o rechazado y, en su caso, las mejoras o modificaciones que se propongan. En el plazo habitual de 2 meses, y máximo de 4, el Consejo Editorial comunicará la aceptación o rechazo de un artículo, junto con las observaciones o sugerencias emitidas por los evaluadores. 
7. Sección de bibliografía. El Consejo Editorial escoge, de entre los libros recibidos en la secretaría de la revista, aquellos que serán objeto de comentario bibliográfico o de recensión. El Consejo Editorial, asimismo, designa, de entre los colaboradores fijos de la sección de bibliografía, a quien haya de encargarse de elaborar el comentario o la recensión. La extensión del comentario bibliográfico se fija en 4.000-6.000 palabras; de la recensión en 1.000-2.000 palabras.

\section{Responsabilidades éticas}

8. Es responsabilidad y deber de la redacción de la revista Ius Canonicum recordar a los autores estos extremos:

- La revista no acepta material previamente publicado. Los autores son responsables de obtener los oportunos permisos para reproducir parcialmente material (texto, tablas o figuras) de otras publicaciones y de citar su procedencia correctamente.

- Conflicto de intereses. La revista espera que los autores declaren cualquier posición o actividad que pueda suponer un conflicto de intereses en conexión con el artículo remitido.

- Autoría. En la lista de autores firmantes deben figurar únicamente aquellas personas que han contribuido intelectualmente al desarrollo del trabajo. En general, para figurar como autor se deben cumplir los siguientes requisitos:

1. Haber participado en la concepción y realización del trabajo que ha dado como resultado al artículo en cuestión.

2. Haber participado en la redacción del texto y en las posibles revisiones del mismo.

La Revista declina cualquier responsabilidad sobre posibles conflictos derivados de la autoría de los trabajos que se publican en la Revista.

- Los juicios y opiniones expresados en los artículos publicados en la Revista son del autor(es) y no necesariamente del Consejo Editorial.

\section{Modos de citar}

9. Libros: J. Hervada, Elementos de Derecho constitucional canónico, Eunsa, Pamplona ${ }^{3} 2014,288$ pp.

O. Condorelli, Unum corpus, diversa capita. Modelli di organizzazione e cura pastorale per una varietas ecclesiarum (secoli XI-XV), Il Cigno Galilei, Roma 2002, 243 pp.

Artículos de revista: A. MORENO GARCÍA, El servicio de indagación prejudicial: aspectos jurídico-pastorales, Ius Canonicum 56 (2016) 65-85.

J.-P. SchoupPe, La dimension juridique de la Parole et des sacrements ainsi que de la «communio», L'année canonique 42 (2000) 167-188. 
Participaciones en obras colectivas: H. PREE, Die Ausübung der Leitungsvollmacht, en J. LISTL - H. MÜLLER - H. SCHMITZ (eds.), Handbuch des katholischen Kirchenrechts, Pustet, Regensburg 1983, 131-141.

Comentarios legislativos: C. Gullo, sub c. 1476, en Comentario exegético al Código de Derecho Canónico, V, Eunsa, Pamplona 32002, 1022-1024.

Voces de diccionario: R. PUZA, «Institutionensystem», en A. V. CAMPENHAUSEN - I. RIEDEL SPANGENBERGER - R. SEbotT (eds.), Lexicon für Kirchen-und Staatskirchenrecht, II, Ferdinand Schöningh, Padeborn-München-Wien-Zürich 2002, 305-307.

D. Cenalmor, «Dimisorias [Letras]», en J. Otaduy - A. Viana - J. Sedano (eds.), Diccionario General de Derecho Canónico, III, Thomson Reuters Aranzadi, Cizur Menor (Navarra) 2013, 335-338 (en adelante, DGDC).

Obras ya citadas: E. Molano, Estructuras jerárquicas..., cit., 327-335.

En las citas deberá evitarse un número excesivo de abreviaturas, limitándose a las más conocidas (CIC, AAS, etc.).

Criterio para obras ya citadas: Primeras palabras del titulo... (puntos suspensivos), cit., 832.

\section{Numeración de títulos y epígrafes}

10. Los títulos de los apartados y subapartados de los Estudios, Comentarios y Crónicas seguirán el siguiente orden y diseño:

1. El OBJETO DE LA EXCLUSióN

\subsection{Planteamiento}

1.1.1. Actos

11. Los epígrafes para los sumarios de los Comentarios y Crónicas seguirán el siguiente orden y diseño:

SUMARIO: 1. Curia romana. 1.1. Congregaciones. 1.1.1. Congregación para la Doctrina de la Fe (modificaciones al motu proprio «Sacramentorum sanctitatis tutela»).

\section{Revisores}

12. - Los artículos se someterán a doble revisión anónima por expertos ajenos al Consejo Editorial.

- Se garantiza la confidencialidad de los autores y de los revisores.

- Periódicamente se dará a conocer la lista de revisores de Ius Canonicum, sin indicación de los trabajos revisados. 



\section{IUS CANONICUM}

DICIEMBRE 2018

VOLUMEN 58

NÚMERO 116

REVISTA SEMESTRAL FUNDADA EN 1961

EDITA: SERVICIO DE PUBLICACIONES DE LA UNIVERSIDAD DE NAVARRA

PAMPLONA / ESPAÑA

ISSN: 0021-325X

\section{A PROPÓSITO DEL SÍNODO SOBRE LOS JÓVENES 2018}

Discernir la idoneidad para el presbiterado: La contribución del derecho y de la tradición canónica latina al Sínodo de Obispos

Nicolás Álvarez de las Asturias. Págs. 461-478

\section{ESTUDIOS}

¿Una alternativa a la noción de executio potestatis? La separación entre potestas ordinis y officium según Hugo de Amiens y Gerhoch de Reichersberg

Thierry Sol. Págs. 481-502

Chiarimenti pontifici sul "processus brevior". Riflessioni alla luce del Discorso

del 25 november 2017

Massimo del Pozzo. Págs. 503-537

La mayoría de edad como presunción iuris tantum de capacidad en los códigos civiles y canónicos

Jorge Castro Trapote. Págs. 539-580

In merito al problema se i Superiori maggiori degli istituti religiosi di diritto pontificio clericali possano erigere pie fondazioni autonome

Geraldina Boni - Manuel Ganarin. Págs. 581-610

Nuevos movimientos eclesiales. Naturaleza de los carismas, cuestiones jurídicas y límites

Luis Navarro. Págs. 611-634

Unidad y pluralidad en la Iglesia. El pluralismo carismático

Javier Otaduy Guerín. Págs. 635-670

Protección de datos: entre el derecho a la intimidad y la autonomía de las confesiones religiosas. El caso finlandés y el español (a propósito de la Sentencia Jehovan Todistajat del TJUE) Jorge Salinas Mengual. Págs. 671-708

Introducción histórica y canónica al oficio eclesiástico

Antonio Viana. Págs. 709-740

Iglesia y Estado en el constitucionalismo isabelino

Francisco José Zamora García. Págs. 741-780

L'instructio probatoria nel processo penale medievale: osservazioni canoniche sull'ammissione e l'assunzione dei mezzi di prova nei secoli XIII e XIV

Ciro Tammaro. Págs. 781-809

COMENTARIOS Y NOTAS. Págs. 813-856

BIBLIOGRAFÍA. Págs. 857-911 